Submitted to International Journal of Heat and Mass Transfer, June 30, 2016, Revised August 28, 2016

\title{
Perfect mid-infrared absorption by hybrid phonon-plasmon polaritons in hBN/metal grating anisotropic structures
}

\author{
Bo Zhao and Zhuomin M. Zhang*
}

\begin{abstract}
A nanostructure based on periodic metal gratings and hexagonal boron nitride $(\mathrm{hBN})$ films is proposed here. As a phononic two-dimensional (2D) material, hBN has two Reststrahlen bands with natural hyperbolic responses. Magnetic polaritons (MPs) in metal gratings can couple with hyperbolic phonon polaritons (HPPs) in hBN to create hybrid hyperbolic phonon-plasmon polaritons, resulting in perfect or near-perfect absorption. It is found that the majority of the power absorbed by the hybrid polaritons is dissipated in the $\mathrm{hBN}$ film with a unique tunable location-dependent absorption profile. Meanwhile, out of the hyperbolic region, optical phonons in $\mathrm{hBN}$ can couple with MPs in gratings to yield resonance absorption. The radiative properties and dissipation profiles are calculated based on rigorous coupled-wave analysis, which has been extended to include anisotropic materials, to elucidate the mechanisms of the perfect and nearperfect absorption. This work reveals the possibility of using $2 \mathrm{D}$ materials and nanostructures to achieve unique radiative properties for applications such as energy harvesting, radiative cooling, and photodetection.
\end{abstract}

Keywords: hexagonal boron nitride, hybrid phonon-plasmon polaritons, hyperbolic 2D materials, metal gratings, perfect absorption 


\section{Nomenclature}

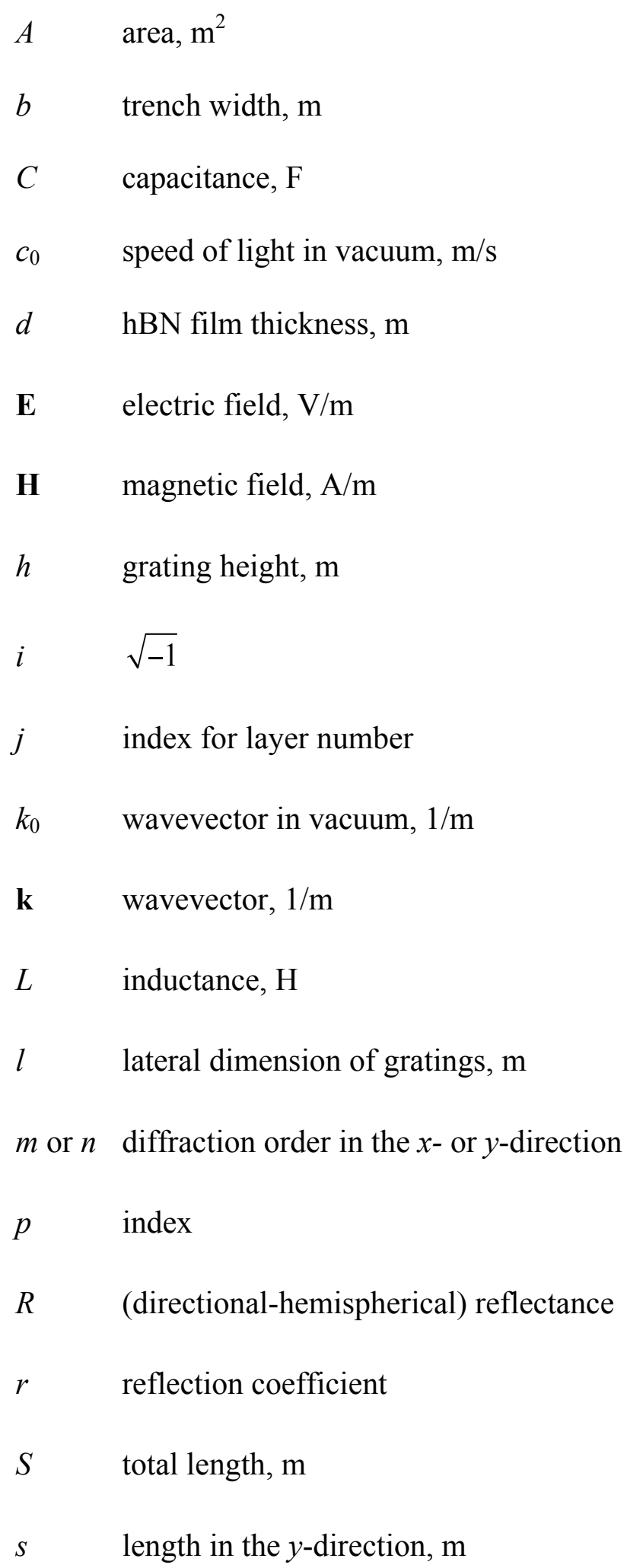




$$
\begin{array}{ll}
t & \text { time, } \mathrm{s} \\
V & \text { volume } \mathrm{m}^{3} \\
w & \text { local power dissipation density, } \mathrm{W} / \mathrm{m}^{3} \\
x, y, z & \text { spatial coordinates, } \mathrm{m} \\
Z & \text { impedance, } \Omega
\end{array}
$$

Greek symbols

$$
\begin{aligned}
& \alpha \quad \text { directional (spectral) absorptance } \\
& \beta \quad \text { propagation angle, deg } \\
& \gamma \quad \text { variable defined in Eq. (5), V/m } \\
& \gamma \quad \text { damping coefficient, } \mathrm{cm}^{-1} \\
& \varepsilon \quad \text { dielectric function } \\
& \varepsilon_{0} \quad \text { permittivity of vacuum, } \mathrm{F} / \mathrm{m} \\
& \theta \quad \text { polar angle, deg } \\
& \Lambda \quad \text { grating period, } \mathrm{m} \\
& \mu_{0} \quad \text { permeability of vacuum, } \mathrm{H} / \mathrm{m} \\
& \sigma \quad \text { index or electrical conductivity, } \mathrm{S} / \mathrm{m} \\
& \phi \quad \text { azimuthal angle, deg } \\
& \chi \quad \text { variable defined in Eq. (5), V/m } \\
& \xi \quad \text { index } \\
& \psi \quad \text { polarization angle, deg }
\end{aligned}
$$


$\omega \quad$ angular frequency, $\mathrm{rad} / \mathrm{s}$

\section{Superscripts}

$\begin{array}{ll}\text { inv } & \text { inverse } \\ \text { ord } & \text { ordinary } \\ r & \text { reflection } \\ t & \text { transmission }\end{array}$

\section{Subscripts}

$\|$ out-of-plane or the $z$-direction

$\perp \quad$ in-plane or the $x$ - or $y$-direction

I, II, III regions defined in Fig. 2

$\begin{array}{ll}\text { Ag } & \text { silver } \\ a, b & \text { indexes } \\ \text { eff } & \text { effective } \\ \text { inc } & \text { incidence } \\ \text { LO longitudinal optical phonon mode } \\ p, q \quad \text { indexes } \\ \text { TO transverse optical phonon mode } \\ \text { tot } \quad \text { total }\end{array}$




\section{Introduction}

Perfect electromagnetic absorption is of critical importance in applications such as energy harvesting, radiative cooling, and photodetection applications [1-8]. Different kinds of metamaterials and nano/microstructures have been proposed to achieve perfect absorption by utilizing various resonance modes. Among them are gratings that can support surface plasmon polaritons (SPPs) $[9,10]$ or surface phonon polaritons $(\mathrm{SPhPs})[11]$, metal/dielectric/metal structures and deep metal gratings that can enable magnetic polaritons (MPs) [12-19], nanoparticles and nanoantennas that can create localized surface plasmon polaritons (LSPPs) [20], and hyperbolic metamaterials that can empower hyperbolic modes and epsilon-near-zero modes [21-23]. These resonances or modes can couple with incident light and capture the energy of the incident waves efficiently [24]. Besides these metamaterials and nanostructures, twodimensional (2D) materials such as graphene and black phosphors offer enormous novel ways to create electromagnetic resonances because of their unique plasmonic properties [25,26]. Various nanostructured resonators and ribbons using 2D materials have been demonstrated to support localized surface plasmon resonances [27-29]. However, with an atomically thin thickness and relatively short light-matter interaction path, it is difficult to achieve perfect absorption in standalone 2D material nanostructures.

A promising way is to combine 2D materials with nano/microstructures. Recent studies suggest that $2 \mathrm{D}$ materials covered nano/microstructures and metamaterials can provide a new route to achieve perfect absorption. Using a hybrid structure with a monolayer graphene covered

on a metal grating, Zhao et al. [30-32] demonstrated strong absorption through excitation of SPPs and MPs. Piper and Fan [33] utilized critical coupling in graphene/photonic crystals hybrid 
structures to achieve perfect absorption. Zhu et al. [34] used a similar mechanism to construct angle-selective perfect absorbers consisting of graphene and a chalcogenide layer atop a mirror. Plasmonic optical nanoantennas with different shapes can also be hybridized with 2D materials to capture light efficiently $[35,36]$. While 2D plasmonic materials and semiconductors have been studied extensively because of their potential applications in microelectronic, optoelectronic, and photonic devices [37], few studies have yet explored the potentials of using phononic 2D materials to achieve perfect absorption through coupling with nano/microstructures, especially for $\mathrm{hBN}$ that is a hyperbolic material. Considering that $\mathrm{hBN}$ can endure a temperature of $1500{ }^{\circ} \mathrm{C}$ in air [38], accomplishing strong absorption or emission bands in the infrared region with $\mathrm{hBN}$ films holds great significance for high-temperature energy harvesting applications [7].

Hyperbolic materials refer to uniaxial materials whose axial (extraordinary) and tangential (ordinary) permittivities have opposite signs. The isofrequency surfaces obey a hyperbolic shape instead of a closed sphere for common isotropic materials. Subsequently, these materials can support propagating modes with very large tangential wavevectors that possess unique applications in sub-wavelength imaging [39-41] and heat transfer [42-45]. Owning to its optical phonon vibrations, hBN naturally possesses two mid-infrared Reststrahlen bands that have hyperbolic response [46,47]. Because of the hyperbolicity, hBN films support multiple orders of low-loss hyperbolic phonon-polariton (HPP) waveguide modes $[48,49]$ that can potentially be utilized to achieve perfect absorption. However, since the lateral wavevectors of these modes are much larger than the magnitude of the wavevector in vacuum, HPPs cannot be excited directly by the incident waves from vacuum. Thus, hybridizing hBN with periodic structures can possibly match the wavevectors required by HPPs and achieve strong absorption induced by phonons. Furthermore, hBN exhibits inverted hyperbolic response since the phonon 
vibrations are in perpendicular crystallographic directions [47], making it an ideal material to explore the basic coupling phenomena between hyperbolic materials and other resonances such as plasmonic polaritons in nano/microstructures.

This work reports a numerical investigation of the possibility to achieve perfect and nearperfect absorption in a hybrid structure consisting of an $\mathrm{hBN}$ film and a metal grating. The strong coupling between HPPs in the hBN film and plasmonic resonances in metal gratings are explored. An anisotropic rigorous-coupled wave analysis (RCWA) algorithm is implemented to calculate the absorptance of the hybrid structure and the local power dissipation density. The geometric effects are evaluated to further elucidate the mechanism of the perfect or near-perfect absorption.

\section{Numerical modeling and theory}

\subsection{Anisotropic rigorous-coupled wave analysis (RCWA)}

The geometric arrangement of the $\mathrm{hBN} /$ metal grating hybrid structure is illustrated in Fig. 1. The $1 \mathrm{D}$ grating is made of silver $(\mathrm{Ag})$ with a period $\Lambda$ in the $x$-direction and extends infinitely in the $y$-direction. Its trench width is $b$ and height is $h$. The thickness of the covered hBN film is denoted as $d$. The substrate of the grating structure is also Ag and assumed to be semi-infinite. In reality, a Ag film whose thickness is much greater than the photon penetration depth can be deposited on another supporting substrate. Thus, the directional (spectral) absorptance of the structure, $\alpha$, can be calculated by $\alpha=1-R$, where $R$ is the directional-hemispherical reflectance of the whole structure that can be calculated using RCWA.

RCWA has been widely used in modeling the radiative properties of periodic structures. However, most available RCWA algorithms are for isotropic materials only. Built on the success

of the isotropic RCWA for one-dimensional (1D) gratings first proposed in 1981 [50], several 
groups expended the formulation to include to anisotropic materials beginning in the 1990s. Glytsis and Gaylord [51] formulated RCWA for 1D anisotropic gratings, but the algorithm may have convergence issues for highly conducting metal gratings because of the way that Fourier factorizations were calculated. The algorithm with the correct Fourier factorization rules was later reformulated by $\mathrm{Li}[52,53]$. Continuous efforts have been devoted and more general algorithms have been formulated to consider 2D arbitrary lattice configurations and permittivity tensor [54-56]. However, most of these works are intended to solve impressively general problems and thus are very complex and formidable to be implemented. The present study provides an easy-to-implement algorithm that allows the modeling of a $2 \mathrm{D}$ multilayered periodic structure made of biaxial materials with a diagonal permittivity tensor. This algorithm is an extension of the public accessible isotropic RCWA code developed by the authors' group [57]. The key features are summarized in the following with selected equations to emphasize the differences from the original RCWA.

Figure 2 illustrates the numerical model of a general 2D anisotropic multilayered periodic structure, which is a more general case than the structure shown in Fig. 1. The periodicity is characterized the periods in the $x$ - and $y$-directions, $\Lambda_{x}$ and $\Lambda_{y}$, respectively. Each layer in the structure can be a 2D pattern/grating, 1D grating or a continuous film by adjusting the lateral dimensions $l_{x}$ and $l_{y}$ as long as $l_{x} \leq \Lambda_{x}$ and $l_{y} \leq \Lambda_{y}$. In the schematic, the first layer is a $2 \mathrm{D}$ grating and the rest are thin films. The medium where the wave is incident from, the intermediate layers (total $N$ layers), and the semi-infinite substrate can be categorized as Region I, II, and III, respectively, as indicated in the schematic. The structure in Fig. 1 is a special case that has a periodicity only in the $x$-direction and only one grating layer in Region II. The incident medium is usually vacuum or a lossless dielectric and thus is treated as isotropic with a dielectric function 
$\varepsilon_{\mathrm{I}}$. The incident wave is assumed to be linearly polarized plane wave with an electric field $\mathbf{E}_{\text {inc }}$. The plane of incidence indicated in transparent gray color is the plane defined by the incident wavevector $\mathbf{k}_{\text {inc }}=\left(k_{x, \text { inc }}, k_{y \text {,inc }}, k_{z \text {,inc }}\right)$ and the $z$-axis. A polar angle $\theta$ (the angle between $\mathbf{k}_{\text {inc }}$ and the $z$-axis ) and azimuthal angle $\phi$ (the angle between the $x$-axis and the plane of incidence) are used to depict the direction of $\mathbf{k}_{\text {inc }}$. Polarization angle $\psi$ is defined as the angle between the electric field and the plane of incidence.

In Region I, the electric field is a superposition of the incident and reflected fields, expressed in the same way as in the isotropic RCWA [9], viz.

$$
\mathbf{E}_{\mathrm{I}}=\mathbf{E}_{\mathrm{inc}} \exp \left(i k_{x, \mathrm{inc}} x+i k_{y, \text { inc }} y+i k_{z, \mathrm{inc}} z\right)+\sum_{m} \sum_{n} \mathbf{E}_{m n}^{\mathrm{r}} \exp \left(i k_{x, m} x+i k_{y, n} y-i k_{z, m n}^{r} z\right)
$$

The time-harmonic term, $\exp (-i \omega t)$, with $\omega$ being the angular frequency, is omitted hereafter. The second term on the right-hand side of Eq. (1) is the summation of the reflected waves. Note that $\mathbf{E}_{m n}^{\mathrm{r}}$ is the complex amplitude of the $(m, n)$ order reflected wave whose transverse wavevector components are determined by the Bloch-Floquet condition [51]:

$$
\begin{aligned}
& k_{x, m}=k_{x, \text { inc }}+m \frac{2 \pi}{\Lambda_{x}} \\
& k_{y, n}=k_{y, \text { inc }}+n \frac{2 \pi}{\Lambda_{y}}
\end{aligned}
$$

where $m$ and $n$ denote the diffraction orders in the $x$ - and $y$-directions, respectively, and they can take both positive and negative integers. The $z$-component of the $(m, n)$ order wavevector is

$$
k_{z, m n}^{r}= \begin{cases}\sqrt{k_{\mathrm{I}}^{2}-k_{x, m}^{2}-k_{y, n}^{2}} \quad, \quad \varepsilon_{\mathrm{I}} k_{0}^{2} \geq k_{x, m}^{2}+k_{y, n}^{2} \\ i \sqrt{k_{x, m}^{2}+k_{y, n}^{2}-k_{\mathrm{I}}^{2}} \quad, \quad \varepsilon_{\mathrm{I}} k_{0}^{2}<k_{x, m}^{2}+k_{y, n}^{2}\end{cases}
$$


where $k_{0}=\omega / c_{0}$ is the wavevector (magnitude) in vacuum with $c_{0}$ being the speed of light in vacuum.

In each layer of Region II, both the electromagnetic field and the dielectric function are expressed as Fourier series based on the periods in the $x$ - and $y$-directions. The materials of each layer are assumed to be nonmagnetic and thus the dielectric function of the layer can be described by a location-dependent permittivity tensor

$$
\overline{\overline{\varepsilon_{j}}}(x, y)=\left(\begin{array}{ccc}
\varepsilon_{j, x} & 0 & 0 \\
0 & \varepsilon_{j, y} & 0 \\
0 & 0 & \varepsilon_{j, z}
\end{array}\right)
$$

where $j=1,2, . . N$ is an index denoting the layer number. Generally, $\varepsilon=\varepsilon^{\prime}+i \varepsilon^{\prime \prime}$ is a complex number with $\varepsilon^{\prime}$ and $\varepsilon^{\prime \prime}$ being its real and imaginary part, respectively. The electric and magnetic fields can be expressed as

$$
\begin{aligned}
& \mathbf{E}_{\boldsymbol{\chi}}=\sum_{m} \sum_{n}{ }_{m n}(z) \exp \left(i k_{x, m} x+i k_{y, n} y\right) \\
& \mathbf{H}_{\boldsymbol{Y}}=i \sqrt{\frac{\varepsilon_{0}}{\mu_{0}}} \sum_{m} \sum_{n} m n(z) \exp \left(i k_{x, m} x+i k_{y, n} y\right)
\end{aligned}
$$

The unknowns $\chi_{m n}(z)$ and $\gamma_{m n}(z)$ can be related by the following equations based on Maxwell's equations, $\nabla \times \mathbf{E}_{\mathrm{II}}-i \omega \mu_{0} \mathbf{H}_{\mathrm{II}}=0$ and $\nabla \times \mathbf{H}_{\mathrm{II}}+i \omega \varepsilon_{0} \overline{\bar{\varepsilon}} \mathbf{E}_{\mathrm{II}}=0$, where $\mu_{0}$ and $\varepsilon_{0}$ are the permeability and permittivity of vacuum, respectively: 


$$
\begin{aligned}
& \frac{\partial \chi_{y, m n}}{\partial z}=\frac{k_{y, n}}{k_{0}} \sum_{p} \sum_{q}\left(\frac{k_{x, p} \gamma_{y, p q}-k_{y, q} \gamma_{x, p q}}{\varepsilon_{z, m-p, n-q}^{\text {ord }}}\right)+k_{0} \gamma_{x, m n} \\
& \frac{\partial \chi_{x, m n}}{\partial z}=\frac{k_{x, m}}{k_{0}} \sum_{p} \sum_{q} \frac{\left(k_{x, p} \gamma_{y, p q}-k_{y, q} \gamma_{x, p q}\right)}{\varepsilon_{z, m-p, n-q}^{\text {ord }}}-k_{0} \gamma_{y, m n} \\
& \frac{\partial \gamma_{y, m n}}{\partial z}=\frac{k_{y, n}}{k_{0}}\left(k_{x, m} \chi_{y, m n}-k_{y, n} \chi_{x, m n}\right)-\sum_{p} \sum_{q} \frac{k_{0} \chi_{x, p q}}{\varepsilon_{x, m-p, n-q}^{\text {inv }}} \\
& \frac{\partial \gamma_{x, m n}}{\partial z}=\frac{k_{x, m}}{k_{0}}\left(k_{x, m} \chi_{y, m n}-k_{y, n} \chi_{x, m n}\right)-\sum_{p} \sum_{q} \frac{k_{0} \chi_{y, p q}}{\varepsilon_{y, m-p, n-q}^{\text {inv }}}
\end{aligned}
$$

where $p$ and $q$ are the summation indices. The superscripts ord and inv indicate the coefficients of the Fourier series for $\underset{\bar{\varepsilon}}{\bar{\varepsilon}}$ and its inverse, respectively, and the expressions are similar to those presented in [58]. The inverse of the dielectric function is used for the sake of fast and guaranteed convergence according to the Fourier factorization rule [52]. Note that different dielectric function components are used in Eq. (6) to deal with anisotropic (uniaxial or biaxial) materials. When all three components of the dielectric tensor are equal, Eq. (6) degenerates to the isotropic scenario presented in [58].

The substrate (Region III) in general can be a biaxial medium. A transmitted plane wave (forward-propagating wave) in this region with an parallel wavevector $\left(k_{x, m}, k_{y, n}\right)$ can have two different $k_{z, m n}^{t}$. If the electric field is $\mathbf{E}_{m n}^{t}=\left(E_{x, m n}^{t}, E_{y, m n}^{t}, E_{z, m n}^{t}\right)$, then based on Maxwell's equations [59], one obtains

$$
\left(\begin{array}{ccc}
\varepsilon_{\mathrm{III}, x} k_{0}^{2}-k_{y, n}^{2}-\left(k_{z, m n}^{t}\right)^{2} & k_{x, m} k_{y, n} & k_{x, m} k_{z, m n}^{t} \\
k_{x, m} k_{y, n} & \varepsilon_{\mathrm{III}, y} k_{0}^{2}-k_{x, m}^{2}-\left(k_{z, m n}^{t}\right)^{2} & k_{y, n} k_{z, m n}^{t} \\
k_{x, m} k_{z, m n}^{t} & k_{y, n} k_{z, m n}^{t} & \varepsilon_{\mathrm{III}, z} k_{0}^{2}-k_{x, m}^{2}-k_{y, n}^{2}
\end{array}\right)\left(\begin{array}{c}
E_{x, m n}^{t} \\
E_{y, m n}^{t} \\
E_{z, m n}^{t}
\end{array}\right)=0
$$


To have nontrivial plane-wave solutions, the determination of the matrix has to be zero and, subsequently, four solutions can be obtained. Two solutions of $k_{z, m n}^{t}$ that correspond to the two forward propagating waves [53] are used to express the electric field in Region III as

$$
\mathbf{E}_{\mathrm{III}}=\sum_{m} \sum_{n} \sum_{\sigma=1,2} E_{\sigma, m n}^{t} \mathbf{p}_{\sigma, m n} \exp \left(i k_{x, m} x+i k_{y, n} y+i k_{\sigma, z, m n}^{t} z\right)
$$

in which

$$
\mathbf{p}_{\sigma, m n}=N_{\sigma, m n}\left(\begin{array}{c}
\left(\varepsilon_{\mathrm{III}, y} k_{0}^{2}-k_{x, m}^{2}-\left(k_{\sigma, z, m n}^{t}\right)^{2}\right)\left(k_{0}^{2} \varepsilon_{\mathrm{III}, z}-k_{x, m}^{2}-k_{y, n}^{2}\right)-k_{y, n}^{2}\left(k_{\sigma, z, m n}^{t}\right)^{2} \\
k_{x, m} k_{y, n}\left(k_{\sigma, z, m n}^{t}\right)^{2}-k_{x, m} k_{y, n}\left(\varepsilon_{\mathrm{III}, z} k_{0}^{2}-k_{x, m}^{2}-k_{y, n}^{2}\right) \\
k_{x, m} k_{y, n}^{2} k_{\sigma, z, m n}^{t}-k_{x, m} k_{\sigma, z, m n}^{t}\left(\varepsilon_{\mathrm{III}, y} k_{0}^{2}-k_{x, m}^{2}-\left(k_{\sigma, z, m n}^{t}\right)^{2}\right)
\end{array}\right)
$$

is the polarization vector for the electric field with diffraction order $(m, n)$ and $\sigma=1$ or 2 is the index for the two forward propagating waves. Note that $N_{\sigma, m n}$ is a coefficient that normalizes $\mathbf{p}_{\sigma, m n}$.

The magnetic field for Region I and III can be obtained from the electric field based on the Maxwell's equation. The complex coefficients in Eq. (5) are then solved through matrix manipulations by matching the tangential component of the electric and magnetic field between adjacent layers. Once the coefficients are solved, the reflectance, transmittance, as well as the field distributions in each layer can be obtained. Note that if the substance is isotropic like the structure to be discussed in this work, the field expression in Eq. (8) will be the same as for isotropic RCWA [58]. In this case, the only difference between the isotropic and anisotropic RCWA exists in Eq. (6). A MATLAB code has been developed based on the above-discussed algorithm. A test case was conducted using a structure shown in Fig. 1 with $\Lambda=4 \mu \mathrm{m}$, $b=300 \mathrm{~nm}, h=1.76 \mu \mathrm{m}$, and $d=30 \mathrm{~nm}$. The structure is $1 \mathrm{D}$ and thus diffraction order $n$ is set 
to zero. The maximum $m$ is set to 71 based on a convergence test and the calculated absorptance spectrum agrees with that calculated using a finite-difference time-domain (FDTD) commercial software (Lumerical Solutions, Inc.). However, RCWA is at least one order of magnitude faster than the FDTD software to model 1D grating structures [9].

The absorptance obtained from $1-R$ is for the whole structure including the hBN film and the Ag grating. To illustrate the local absorption profile inside the structure, the local power dissipation density (in $\mathrm{W} / \mathrm{m}^{3}$ ) can be calculated based on [60]

$$
w(x, y, z)=\frac{1}{2} \operatorname{Re}\left\{-i \omega \varepsilon_{0}=\underset{\varepsilon}{\bar{E}} \mathbf{E} \mathbf{g}^{*}\right\}=\frac{1}{2} \varepsilon_{0} \omega\left(\varepsilon_{x}^{\prime \prime}\left|E_{x}\right|^{2}+\varepsilon_{y}^{\prime \prime}\left|E_{y}\right|^{2}+\varepsilon_{z}^{\prime \prime}\left|E_{z}\right|^{2}\right)
$$

in which $\mathbf{E}$ is the complex electric field obtained from RCWA. The absorptance of a certain volume or layer can be calculated by the ratio of the absorption inside the volume over the incident power [30,31]:

$$
\alpha=\frac{\iint \oint(x, y, z) d V}{0.5 c_{0} \varepsilon_{0}\left|\mathbf{E}_{\text {inc }}\right|^{2} A \cos \theta}
$$

The denominator is the incident power on area $A$ at a polar angle $\theta$. Using Eq. (11), the absorptance inside the hBN film and the grating can be separately retrieved.

\subsection{Hyperbolic phonon-polaritons in hBN films}

The real and imaginary parts of the dielectric function of $\mathrm{hBN}$ are shown in Fig. 3. The two mid-infrared Reststrahlen bands due to the optical phonon modes are evident. The in-plane phonon modes $\left(\omega_{\mathrm{TO}, \perp}=1370 \mathrm{~cm}^{-1}\right.$ and $\left.\omega_{\mathrm{LO}, \perp}=1610 \mathrm{~cm}^{-1}\right)$ and out-of-plane phonon modes ( $\omega_{\mathrm{TO}, \mathbf{P}}=780 \mathrm{~cm}^{-1}$ and $\left.\omega_{\mathrm{LO}, \mathbf{P}}=830 \mathrm{~cm}^{-1}\right)$ contribute to the in-plane $(\mathbf{E}$ lies in the $x-y$ plane, 
denoted by $\perp$ ) and out-of-plane ( $\mathbf{E}$ parallel to the optical axis or the $z$-direction, denoted by $\|$ ) dielectric functions, respectively [49]:

$$
\varepsilon_{\xi}=\varepsilon_{\infty, \xi}\left(1+\frac{\omega_{\mathrm{LO}, \xi}^{2}-\omega_{\mathrm{TO}, \xi}^{2}}{\omega_{\mathrm{TO}, \xi}^{2}-i \gamma_{\xi} \omega-\omega^{2}}\right)
$$

where $\xi=\mathbf{P}, \perp$. The other parameters used are $\varepsilon_{\infty, \mathbf{P}}=2.95, \gamma_{\mathbf{P}}=4 \mathrm{~cm}^{-1}, \varepsilon_{\infty, \perp}=4.87$, and $\gamma_{\perp}=5 \mathrm{~cm}^{-1}$. Since the damping coefficients $\gamma$ are rather small, the dielectric function becomes negative between the TO and LO phonon modes, making the in-plane and out-of-plane dielectric functions of $\mathrm{hBN}$ possess opposite signs in either Reststrahlen band. In the lower-frequency Reststrahlen band, $\varepsilon_{\mathbf{P}}^{\prime}=\varepsilon_{z}^{\prime}<0$ and $\varepsilon_{\perp}^{\prime}=\varepsilon_{x}^{\prime}=\varepsilon_{y}^{\prime}>0$, hBN has Type-I hyperbolicity, while in the higher-frequency Reststrahlen band, $\varepsilon_{x}^{\prime}=\varepsilon_{y}^{\prime}<0$ and $\varepsilon_{z}^{\prime}>0$, hBN holds Type-II hyperbolicity [47]. Meanwhile, in the two regions with hyperbolicity shown by the shaded areas on Fig. 3, loss is negligibly small except near the band edge as indicated by the imaginary part.

The hyperbolic regions allow waves with unbounded wavevectors to propagate as can be seen from the isofrequency surface for an uniaxial medium whose optical axis lies in the $z$ direction [45]:

$$
\frac{k_{x}^{2}+k_{y}^{2}}{\varepsilon_{\mathbf{P}}}+\frac{k_{z}^{2}}{\varepsilon_{\perp}}=\frac{\omega^{2}}{c_{0}^{2}}
$$

where $\mathbf{k}=\left(k_{x}, k_{y}, k_{z}\right)$ represents the allowed wavevector. If loss is neglected, at frequencies where the material possesses hyperbolicity, Eq. (13) becomes a hyperboloid and hence both $k_{x}$ and $k_{z}$ can theoretically be infinitely large. Note that Eq. (13) is for extraordinary waves or transverse magnetic (TM) waves, which are the primary interest here. For transverse electric (TE) 
waves, the isofrequency surface becomes a sphere described by $|\mathbf{k}|^{2}=\varepsilon_{\perp} \omega^{2} / c_{0}^{2}$, thus hBN behaves the same as an isotropic material with the ordinary dielectric function. For $k_{y}=0$, Eq. (13) can be drawn in $k_{x}-k_{z}$ plane as shown in Fig. 4. For both Type-I and Type-II regions, at very large $|\mathbf{k}|$, the allowed wavevectors approach the asymptotic lines described by $k_{z}= \pm k_{x} \sqrt{-\varepsilon_{\perp} / \varepsilon_{\mathbf{P}}}$ (neglecting loss). In this case, the Poynting vector (i.e., energy propagation direction of the polaritons) is orthogonal to the isofrequency curve. The polarition propagating angle is defined as the angle between the Poynting vector and the $z$-axis and is approximately equal to $[39-41]$

$$
\beta(\omega)=\arctan \left(\sqrt{-\frac{\varepsilon_{\perp}}{\varepsilon_{\mathbf{P}}}}\right)
$$

Equation (14) suggests that at a given frequency, there exists a unique propagation direction inside the hyperbolic material at sufficiently large lateral wavevectors.

For an hBN film, HPPs are supported in the two Reststrahlen bands or hyperbolic regions. Their dispersion can be obtained from the reflection coefficient of TM waves for an $\mathrm{hBN}$ film of a thickness $d$ suspended in vacuum:

$$
r=\frac{r_{12}+\left(1-r_{12}-r_{21}\right) r_{23} \exp \left(2 i k_{z, 2} d\right)}{1-r_{21} r_{23} \exp \left(2 i k_{z, 2} d\right)}
$$

where indexes 1 and 3 are vacuum and 2 indicates the hBN film. The reflection coefficients between the vacuum and $\mathrm{hBN}$ or vice versa are given by

$$
r_{a b}=\frac{\frac{\varepsilon_{\perp, b}}{k_{z, b}}-\frac{\varepsilon_{\perp, a}}{k_{z, a}}}{\frac{\varepsilon_{\perp, b}}{k_{z, b}}+\frac{\varepsilon_{\perp, a}}{k_{z, a}}}
$$


where $a=1$ or 2 and $k_{z, b}=\left(\varepsilon_{\perp, b} k_{0}^{2}-\varepsilon_{\perp, b} \beta^{2} / \varepsilon_{\mathbf{P}, b}\right)^{1 / 2}$ with $b$ being 1,2 or 3 is the $z$-component of the wavevector in $\mathrm{hBN}$ or vacuum. For vacuum, one can set $\varepsilon_{\perp}=\varepsilon_{\mathbf{P}}=1$. The poles of Eq. (15) indicate the dispersion of HPPs in hBN films that can be seen by the bright bands from the contour plots of the imaginary part of $r$ in $k_{x}-\omega$ space [49], as shown in Figs. 5(a) and (b) for a 30 nm-thick hBN film in the frequency ranges near the higher and the lower hyperbolic regions, respectively. Multiple orders of HPPs exist in both hyperbolic regions and more orders are excited with thicker $\mathrm{hBN}$ films. Alternatively, when $k_{x}>k_{0}$, the dispersion based on $1-r_{21} r_{23} \exp \left(2 i k_{z, 2} d\right)=0$ can be approximated by [48]

$$
k_{x}(\omega)=\frac{1}{d} \sqrt{-\frac{\varepsilon_{\mathbf{P}}}{\varepsilon_{\perp}}}\left[p \pi \pm 2 \arctan \left(\frac{1}{\sqrt{-\varepsilon_{\mathbf{P}} \varepsilon_{\perp}}}\right)\right]
$$

where integer $p$ indicates the order of the HPPs. The plus and minus signs are chosen respectively for the higher and lower hyperbolic bands based on the shape of the dispersion curves [49]. The prediction from Eq. (17) is overlaid on the contour plot as broken lines in Fig. 5 with the corresponding $p$. Note that $p=1$ yields negative $k_{x}$ in the lower hyperbolic region and thus is not shown. Clearly, Eq. (17) provides a convenient description of HPPs and will be referred to in later discussions. Since the HPPs have sufficient large wavevectors, Eq. (14) is valid and thus different orders of HPPs at the same frequency have the same energy flux propagation direction, as will be further demonstrated.

\section{Results and Discussion}

\subsection{Perfect and near-perfect absorption}


Figure 6 shows the absorptance spectra of plain gratings (dashed lines) and hBN-covered Ag gratings (solid lines) calculated based on the anisotropic RCWA algorithm for TM waves. Unless specified otherwise, the wave is incident normal to the $x-y$ plane and the plane of incidence is set as the $x-z$ plane. Since the considered structures are one-dimensional gratings and the electric field for TM waves is in the $x$-direction; therefore, $\theta=\psi=\phi=0^{\circ}$ and $k_{y}=0$. The dielectric function of Ag is calculated using a Drude model [14]. Throughout the modeling, the parameters are set as $\Lambda=4 \mu \mathrm{m}, b=300 \mathrm{~nm}$, and $d=30 \mathrm{~nm}$, while $h$ are allowed to change. Such grating geometries can create excitations of MPs in the infrared region as demonstrated before [14], but $\Lambda$ and $b$ can be other values to make MPs in the infrared. In Figs. 6(a), (b), and (c), $h$ is $1.76 \mu \mathrm{m}, 2.4 \mu \mathrm{m}$, and $0.88 \mu \mathrm{m}$, respectively. One absorptance peak can be identified on each plain Ag grating spectrum, and this peak is caused by the excitation of MP resonances [14]. MPs are formed by the coupling of the local resonance inside a micro/nanostructure and the external electromagnetic waves [14,61]. Upon excitation, MP resonance induces an oscillating current loop that can result in strong dissipation, as indicated by the absorptance peaks. In Ag gratings, a high absorptance peak due to MP relies on an efficient coupling between the surface waves on the trench walls that can be achieved only when the trench is very narrow [14]. Thus, for trench width $b=300 \mathrm{~nm}$, the absorptance peaks for plain Ag gratings are far from unit in Fig. 6. High absorptance is difficult to achieve with thin $\mathrm{hBN}$ films either, as demonstrated by the absorptance spectrum of a suspended 30-nm hBN film in Fig. 6(a). Only a small absorptance peak at $\omega_{\mathrm{TO}, \perp}$ is obtained, since no HPPs can be excited with propagating waves in air. However, after covering the $\mathrm{hBN}$ film on the $\mathrm{Ag}$ grating, perfect absorption $(\alpha=1)$ and near-perfect absorption $(\alpha=0.99)$ can be achieved at $v=1436 \mathrm{~cm}^{-1}$ and $v=818.7 \mathrm{~cm}^{-1}$ as shown in Figs. 6(a) and (b), respectively. These two high absorptance peaks fall in the hyperbolic regions of $\mathrm{hBN}$, 
while the absorption peak ( $\alpha=0.94)$ at $v=1333 \mathrm{~cm}^{-1}$, as shown in Fig. $6(\mathrm{c})$, is slightly below the higher-frequency hyperbolic region. High absorptance or emittance has important applications in thermophotovoltaics, photodetection, and radiative cooling. The origin of these high absorption peaks and other peaks on the spectra will be elucidated in the following.

Figures 7(a) and (b) exhibit the absorptance contours for plain and hBN-covered gratings, respectively, with respect to the wavenumber and grating height. Two bright branches due to excitation of MPs show up in Fig. 7(a). The fundamental MP modes, corresponding to the lower branch, can be predicted using the inductor-capacitor (LC) circuit model [62-64] by considering inductance from the grating trench, $L_{\mathrm{Ag}}$, and capacitance from the vacuum region inside the trench, $C$, as has been previously investigated [14]. The resonance condition can be obtained from the natural frequency of the equivalent LC circuit by zeroing the imaginary part of the total impedance. The predicted resonance conditions are shown in Fig. 7(a) as round markers, giving a good agreement with RCWA calculation. The upper branch correspond to higher-order MP and will not be discussed further [65]. After the hBN is covered as seen in Fig. 7(b), the fundamental resonance branch has a noticeably different shape compared to Fig. 7(a). A disconnection close to $\omega_{\mathrm{TO}, \perp}$ occurs and high absorptance is achieved inside the two hyperbolic regions as well as at frequencies that are slightly lower than $\omega_{\mathrm{TO}, \perp}$. The spectra presented in Fig. 6 are representatives of the high absorptance in different frequency ranges achieved at specific grating heights. Note that the hyperbolic regions are indicated between two white horizontal lines. The fundamental modes can be predicted by adding $Z_{\mathrm{hBN}}$ in the LC circuit, as shown in Fig. 1 . However, $Z_{\mathrm{hBN}}$ takes different formula in different frequency ranges. 
The frequency-dependent impedance of $\mathrm{hBN}$ can be obtained from $Z_{\mathrm{hBN}}=S /\left(\sigma A_{\mathrm{eff}}\right)$, where $S$ is the length along the current path, $\sigma=-i \omega \varepsilon \varepsilon_{0}$ is the electrical conductivity, and $A_{\text {eff }}$ is the effective cross-section area [61]. Similar to the case for graphene-covered Ag gratings [30,31], the hybrid structure possess an electric field in the trench that is very similar to the electric field when MPs are excited in plain gratings. Thus, only the part of $\mathrm{hBN}$ that across the trench opening participates in the LC circuit, i.e., $S=b$. The penetration depth of the electric field in $\mathrm{hBN}$ is much greater than $d$ and thus $A_{\mathrm{eff}}=s d$, where $s$ is the length in the $y$-direction that can be set to unity for one-dimensional gratings. Meanwhile, the electric field near the trench opening in $\mathrm{hBN}$ is dominated by its $x$-component [30,31], indicating that the conductivity should be evaluated based $\varepsilon_{\perp}$ of $\mathrm{hBN}$. The real part of $Z_{\mathrm{hBN}}$ is the resistance and it is negligibly small in the frequency regions of interest. The imaginary part of $Z_{\mathrm{hBN}}$ can be positive or negative since $\varepsilon_{\perp}^{\prime}$ takes different signs depending on the frequency range, meaning that $\mathrm{hBN}$ can serve as a capacitor or inductor $[62,63]$. In the upper hyperbolic region, $\varepsilon_{\perp}^{\prime}$ is negative due to the inplane phonon vibrations as shown in Fig. 3(a), and hBN acts like an inductor across the trench opening with an inductance

$$
L_{\mathrm{hBN}}=-\frac{\varepsilon_{\perp}^{\prime} b}{\varepsilon_{0}\left|\varepsilon_{\perp}\right|^{2} \omega^{2} s d}
$$

On the other hand, outside the higher-frequency hyperbolic region, $\varepsilon_{\perp}^{\prime}$ is positive and hBN provides a capacitance

$$
C_{\mathrm{hBN}}=\frac{\varepsilon_{0} s d\left|\varepsilon_{\perp}\right|^{2}}{\varepsilon_{\perp}^{\prime} b}
$$

Equations (18) and (19) can be obtained based on $Z_{\mathrm{hBN}}$. 
After all the elements in the circuit are known, the resonance frequency can be obtained as $v=\left(2 \pi c_{0} \sqrt{L_{\mathrm{tot}} C_{\mathrm{tot}}}\right)^{-1}$ [32]. In the upper hyperbolic region, $L_{\mathrm{hBN}}$ and $L_{\mathrm{Ag}}$ are in parallel, yielding $L_{\mathrm{tot}}=L_{\mathrm{Ag}} L_{\mathrm{hBN}} /\left(L_{\mathrm{Ag}}+L_{\mathrm{hBN}}\right)$ and $C_{\mathrm{tot}}=C$. The total inductance is reduced by $\mathrm{hBN}$ compared to plain gratings and thus the fundamental resonance in the hybrid structure shifts to frequencies higher than those in plain gratings as shown in Fig. 6(a). Outside the hyperbolic region, $C_{\mathrm{hBN}}$ and $C$ are connected in parallel, yielding $L_{\mathrm{tot}}=L_{\mathrm{Ag}}$ and $C_{\mathrm{tot}}=C+C_{\mathrm{hBN}}$. This makes the total capacitance larger, shifting the MP resonance to lower frequencies, as demonstrated in Figs. 6(b) and (c). The LC model (round markers) matches the RCWA simulation very well, as shown in Fig. 7(b). The good agreement justifies that the fundamental resonance is a coupled resonance, which has some unique features depending on the frequency range as discussed next.

\subsection{Hybrid phonon-plasmon polaritons and tunable location-dependent absorption}

Figure 8 shows the local power dissipation profile at the perfect-absorption peak shown in Fig. 6(a), which lies in the high-frequency hyperbolic region, where Fig. 8(a) displays a zoomed-in view of the hBN film and the unit of the contour scale for $w$ is $\mathrm{MW} / \mathrm{m}^{3}$. For Fig. $8(\mathrm{~b})$, $w$ is multiplied by a factor of 10 to show the dissipation near the surface of the Ag groove clearly. For example, at the trench bottom, the actual value is $w \approx 0.019 \mathrm{MW} / \mathrm{m}^{3}$ in $\mathrm{Ag}$. In the calculation, the magnitude of the incident electric field is $1 \mathrm{~V} / \mathrm{m}$. It can be seen that dissipation is mainly at the surface of the grating, similar to previous observations of metal gratings at MP resonances [30-32]. The absorption in the hBN film is much stronger than in the grating. The maximum $w$ in $\mathrm{hBN}$ is $2.4 \mathrm{MW} / \mathrm{m}^{3}$, which exceeds the upper limit of the scale bar. The absorptance of the $\mathrm{hBN}$ 
film can be obtained by integration using Eq. (11) as 0.91 , suggesting that most of the incident power is absorbed by the hBN film instead of the grating.

As can be seen from Fig. 8(a), the dissipation profile follows a zigzag path in the hBN film that is symmetric about the middle of the trench, indicating that the power is nonuniformly absorbed in the film. This dissipation profile is a unique feature of the HPP waveguide modes [39-41]. Due to the diffraction of the grating, electric fields with large wavevectors are generated and they excite the hyperbolic polaritons in the $\mathrm{hBN}$ film. The polaritons predominantly initiate from the two corners of the grating because of the highly concentrated electric field therein that is about 50 times that of the incident waves. The polariton rays propagate inside the film with a fixed angle of $\beta=65.7^{\circ}$ as described by Eq. (14) and experience a total internal reflection on the top surface of the hBN film [40]. The predicted angle matches Fig. 8(a) well, even though loss is neglected Eq. (14). Furthermore, the period of the zigzag pattern in the $x$-direction can be derived as $2 d \tan \beta$ based on the wavevector of the polaritons. It should be noted that multiple orders of HPPs are excited simultaneously at the resonance frequency due to grating diffraction. For a specific frequency, according to Eq. (17), the wavevectors of the multiple HPPs form an arithmetic progression with a common difference $\Delta k_{x}=(\pi / d) \sqrt{-\varepsilon_{\mathbf{P}} / \varepsilon_{\perp}}$. Thus, these HPPs produce an interference pattern with a spatial period $2 \pi / \Delta k_{x}[40]$, which is the same as the above-obtained period in real space, providing a complementary way to understand the periodic zigzag pattern. Due to the weak loss of hBN, these HPPs dissipate power as they propagate inside the film and gradually vanish. The weak loss of $\mathrm{hBN}$ enables a long propagation length that is an advantage of $\mathrm{hBN}$ in subdiffractional focusing and imaging applications over other hyperbolic metamaterials constructed with metallic nanowires or metal/dielectric multilayers 
[39-41]. The unique directional propagation of the polaritons allows multiple reflections and long light-matter interaction distances, giving rise to the strong absorption in $\mathrm{hBN}$.

Figure 9 illustrates a similar local power dissipation profile, which corresponds the highabsorptance resonance at $v=818.7 \mathrm{~cm}^{-1}$ (in the lower hyperbolic region) in Fig. 6(b). The dissipation in $\mathrm{hBN}$ is again much stronger than in $\mathrm{Ag}$, reaching $8 \mathrm{MW} / \mathrm{m}^{3}$ at the two corners of the grating and $84 \%$ of the incident power is dissipated in $\mathrm{hBN}$ with a zigzag dissipation pattern with $\beta=72.4^{\circ}$. Due to higher loss at this resonance, the HPPs can only survive a relatively shorter propagation distance. Based on the above analysis, the dissipation profile at the perfect and near-perfect absorption in hyperbolic regions possesses both features of a MP and HPPs and, thus, these resonances are hybrid hyperbolic phonon-plasmon polaritons formed by strong coupling between plasmonic MP in the metal grating and HPPs in the hBN film.

The scenario of the near-perfect absorption at $v=1333 \mathrm{~cm}^{-1}$ shown in Fig. 6(c) is different from the above discussion since it is not in the hyperbolic regions. Figure 10(a) shows the dissipation profile at this resonance. Here, the absorption in the hBN film is concentrated within the portion that is across the trench opening of the grating. The scale bar is nonlinear beyond $0.5 \mathrm{MW} / \mathrm{m}^{3}$ to show the dissipation profile in the grating is again due to the excitation of MP [30,31]. Using Eq. (11), one can obtain the absorptance of $\mathrm{hBN}$ to be 0.72 , which is more than 3 times of the value in the Ag grating (i.e., 0.22). The main reason for this high absorptance is an enhanced electric field near the trench opening when a MP is excited; this is very similar to the high absorptance in graphene-covered metal gratings [14,30,31]. Since the electric field strength decays gradually away from the trench opening, $w$ becomes progressively smaller in hBN film as illustrated in Fig. 10(b), showing the dissipation profiles at different height in the film. At the bottom of the hBN film, $w$ shows two spikes at the two corners of the grating due to 
the highly concentrated electric field therein. This strong electric field is also important to the zigzag dissipation profile of the hyperbolic phonon-plasmon polaritons as discussed before. Therefore, the strong absorption is a result of hybrid phonon-plasmon polaritons. Here the hBN film provides additional capacitance in the LC circuit that pushes the resonances to lower frequencies.

\subsection{Other effects}

The general effect of the geometric parameters can be understood using the abovementioned LC circuit model. For example, when $d$ is increased from $30 \mathrm{~nm}$, the inductance of hBN calculated from Eq. (18) will decrease in the upper hyperbolic region and push the coupled resonances to higher frequencies, and vice versa in the frequency range that $\mathrm{hBN}$ is a capacitor. However, there exist some flat bright bands in the two hyperbolic regions as shown in Fig. 7(b) that cannot be explained by the LC circuit. These resonances are HPPs excited by the electric field with large wavevectors due to grating diffraction. When HPPs are coupled with the MP in grating at a certain grating height, the hybrid polaritons push the absorptance higher compared to the absorptance of uncoupled hyperbolic polaritons at other grating heights. These flat bands are discrete and become sparser in frequency when the $d$ becomes larger, though not shown here, indicating that the hyperbolic phonon polaritons are excited at specific frequencies instead of the whole hyperbolic regions. This is very different with the scenario where the structure is not periodic since continuous high wavevectors can be created [41]. Therefore, the hBN film thickness, the grating height, as well as the trench width, can all be used to control the excitation condition of the hybrid hyperbolic phonon-plasmon polaritons. Since the propagation angle of the HPPs depends on the frequency, these geometry parameters can be used to manipulate the 
location of the absorption in hBN films. Furthermore, the excitation of hybrid polaritons is insensitive to the incident direction up to about $45^{\circ}$. For example, calculations show that when the incidence angle is changed to $30^{\circ}$, the high-absorptance peaks shown in Figs. 6(a), (b), and (c) shift slightly to $1430 \mathrm{~cm}^{-1}, 819.7 \mathrm{~cm}^{-1}$, and $1331 \mathrm{~cm}^{-1}$, respectively, with the corresponding absorptance being $0.99,0.98$, and 0.93 . The propagation direction of the HPPs of the first two hybrid polaritons is in consistent with the slightly changed resonance frequencies.

Practical experiments may have some discrepancies with the theoretical ideal structure. For example, fabricated metal gratings may not be perfectly flat and leave a vacuum gap between hBN film and grating. However, such an imperfection may not significantly alter the results since no identifiable difference are found in the three spectra shown in Fig. 6 when a 1-nm gap between $\mathrm{hBN}$ and metal gratings is present, though not shown here. Meanwhile, the corners of the fabricated grating may not be as sharp as the ideal case, and thus the electric field would be expected to be somehow weaker around the grating edges. Such gratings may still be able to concentrate an electric field that is strong enough to launch a strong HPP in hBN and achieve high absorption, but the excited polariton may be less distinguishable with lower spatial contrast [41]. The above-discussed unique properties enabled by the hybrid hBN film and grating nano/microstructures have potential applications in sub-wavelength imaging [41] and surfaceenhanced Raman spectroscopy [66]. Considering that gratings made of high-temperature materials like tungsten [9] and $\mathrm{SiC}$ [61] also support MPs, the design presented here could be used to build stable perfect absorbers or spectral selective emitters for high-temperature applications [7].

\section{Conclusions}


This work demonstrates perfect and near-perfect absorption in $\mathrm{hBN} /$ metal grating hybrid anisotropic structures due to hybrid phonon-plasmon polaritons. The hybrid polaritons can be explained with an LC circuit model in which hBN behaves as either an inductor or capacitor depending on the frequency range. In the two hyperbolic regions of hBN, HPPs strongly couple with localized MPs, forming hybrid hyperbolic phonon-plasmon polaritons and boosting the absorptance. The majority of the power is dissipated inside the hBN film with a tunable locationdependent absorption profile. Outside the hyperbolic regions, the optical phonons in $\mathrm{hBN}$ can strongly couple with MPs and hBN dissipates significant power because of its intrinsic loss and the strong electric field produced by MP resonances. The height of the grating, the trench width, as well as the hBN film thickness, can be used to tune the perfect and near-perfect absorption to different frequencies. This work reveals the possibility of using hyperbolic $2 \mathrm{D}$ materials to achieve perfect absorption and sheds light on a new route to construct hybrid structures with unique radiative properties for sub-wavelength imaging and high-temperature energy harvesting.

\section{Acknowledgements}

The research was supported by the National Science Foundation (CBET-1235975; CBET-1603761). 


\section{References}

[1] S. Basu, Y.-B. Chen, and Z.M. Zhang, Microscale radiation in thermophotovoltaic devices - a review. Int. J. Energy Res. 31 (2007) 689-716.

[2] N.I. Landy, S. Sajuyigbe, J.J. Mock, D.R. Smith, and W.J. Padilla, Perfect metamaterial absorber. Phys. Rev. Lett. 100 (2008) 207402.

[3] H.A. Atwater and A. Polman, Plasmonics for improved photovoltaic devices. Nat. Mater. 9 (2010) 205-213.

[4] X. Liu, T. Tyler, T. Starr, A.F. Starr, N.M. Jokerst, and W.J. Padilla, Taming the blackbody with infrared metamaterials as selective thermal emitters. Phys. Rev. Lett. 107 (2011) 045901.

[5] Y. Cui, K.H. Fung, J. Xu, H. Ma, Y. Jin, S. He, and N.X. Fang, Ultrabroadband light absorption by a sawtooth anisotropic metamaterial slab. Nano Lett. 12 (2012) 1443-1447.

[6] A.P. Raman, M.A. Anoma, L. Zhu, E. Rephaeli, and S. Fan, Passive radiative cooling below ambient air temperature under direct sunlight. Nature 515 (2014) 540-544.

[7] I.E. Khodasevych, L. Wang, A. Mitchell, and G. Rosengarten, Micro- and nanostructured surfaces for selective solar absorption. Adv. Opt. Mater. 3 (2015) 852-881.

[8] W. Li and J. Valentine, Metamaterial perfect absorber based hot electron photodetection. Nano Lett. 14 (2014) 3510-3514.

[9] B. Zhao, L. Wang, Y. Shuai, and Z.M. Zhang, Thermophotovoltaic emitters based on a two-dimensional grating/thin-film nanostructure. Int. J. Heat Mass Transfer 67 (2013) 637-645.

[10] C.R. Williams, S.R. Andrews, S.A. Maier, A.I. Fernandez-Dominguez, L. Martin Moreno, and F.J. Garcia-Vidal, Highly confined guiding of terahertz surface plasmon polaritons on structured metal surfaces. Nat. Photonics 2 (2008) 175-179.

[11] J.-J. Greffet, R. Carminati, K. Joulain, J.-P. Mulet, S. Mainguy, and Y. Chen, Coherent emission of light by thermal sources. Nature 416 (2002) 61-64.

[12] B.J. Lee, L.P. Wang, and Z.M. Zhang, Coherent thermal emission by excitation of magnetic polaritons between periodic strips and a metallic film. Opt. Express 16 (2008) 11328-11336. 
[13] H. Wang and L. Wang, Perfect selective metamaterial solar absorbers. Opt. Express 21 (2013) A1078-A1093.

[14] B. Zhao and Z.M. Zhang, Study of magnetic polaritons in deep gratings for thermal emission control. J. Quant. Spectrosc. Radiat. Transfer 135 (2014) 81-89.

[15] Y. Xuan and Y. Zhang, Investigation on the physical mechanism of magnetic plasmons polaritons. J. Quant. Spectrosc. Radiat. Transfer 132 (2014) 43-51.

[16] R. Feng, W. Ding, L. Liu, L. Chen, J. Qiu, and G. Chen, Dual-band infrared perfect absorber based on asymmetric T-shaped plasmonic array. Opt. Express 22 (2014) A335A343.

[17] B. Zhao, A. Sakurai, and Z.M. Zhang, Polarization dependence of the reflectance and transmittance of anisotropic metamaterials. J. Thermophys Heat Transfer 30 (2016) 240246.

[18] Y. Zhao and C. Fu, Numerical simulation on the thermal radiative properties of a $2 \mathrm{~d}$ $\mathrm{SiO}_{2} / \mathrm{W} / \mathrm{SiO}_{2} / \mathrm{W}$ layered grating for thermophotovoltaic applications. J. Quant. Spectrosc. Radiat. Transfer 182 (2016) 35-44.

[19] J. Song, M. Si, Q. Cheng, and Z. Luo, Two-dimensional trilayer grating with a metal/insulator/metal structure as a thermophotovoltaic emitter. Applied Optics 55 (2016) 1284-1290.

[20] B. Luk'yanchuk, N.I. Zheludev, S.A. Maier, N.J. Halas, P. Nordlander, H. Giessen, and C.T. Chong, The fano resonance in plasmonic nanostructures and metamaterials. Nat. Mater. 9 (2010) 707-715.

[21] H.N.S. Krishnamoorthy, Z. Jacob, E. Narimanov, I. Kretzschmar, and V.M. Menon, Topological transitions in metamaterials. Science 336 (2012) 205-209.

[22] X.L. Liu, L.P. Wang, and Z.M. Zhang, Wideband tunable omnidirectional infrared absorbers based on doped-silicon nanowire arrays. J. Heat Transfer 135 (2013) 061602061602.

[23] M.A.K. Othman, C. Guclu, and F. Capolino, Graphene-dielectric composite metamaterials: Evolution from elliptic to hyperbolic wavevector dispersion and the transverse epsilon-near-zero condition. J. Nanophotonics 7 (2013) 073089-073089.

[24] S.V. Boriskina, H. Ghasemi, and G. Chen, Plasmonic materials for energy: From physics to applications. Mater. Today 16 (2013) 375-386. 
[25] F. Xia, H. Wang, D. Xiao, M. Dubey, and A. Ramasubramaniam, Two-dimensional material nanophotonics. Nat. Photonics 8 (2014) 899-907.

[26] F.H.L. Koppens, D.E. Chang, and F.J. García de Abajo, Graphene plasmonics: A platform for strong light-matter interactions. Nano Lett. 11 (2011) 3370-3377.

[27] A. Nikitin, F. Guinea, F. Garcia-Vidal, and L. Martin-Moreno, Surface plasmon enhanced absorption and suppressed transmission in periodic arrays of graphene ribbons. Phys. Rev. B 85 (2012) 081405.

[28] B. Liu, Y. Liu, and S. Shen, Thermal plasmonic interconnects in graphene. Phys. Rev. B 90 (2014) 195411.

[29] Z. Liu and K. Aydin, Localized surface plasmons in nanostructured monolayer black phosphorus. Nano Lett. 16 (2016) 3457-3462.

[30] B. Zhao, J.M. Zhao, and Z.M. Zhang, Enhancement of near-infrared absorption in graphene with metal gratings. Appl. Phys. Lett. 105 (2014) 031905.

[31] B. Zhao, J.M. Zhao, and Z.M. Zhang, Resonance enhanced absorption in a graphene monolayer using deep metal gratings. J. Opt. Soc. Am. B 32 (2015) 1176-1185.

[32] B. Zhao and Z.M. Zhang, Strong plasmonic coupling between graphene ribbon array and metal gratings. ACS Photonics 2 (2015) 1611-1618.

[33] J.R. Piper and S. Fan, Total absorption in a graphene monolayer in the optical regime by critical coupling with a photonic crystal guided resonance. ACS Photonics 1 (2014) 347353.

[34] L. Zhu, F. Liu, H. Lin, J. Hu, Z. Yu, X. Wang, and S. Fan, Angle-selective perfect absorption with two-dimensional materials. Light: Science \& Applications 5 (2016) e16052.

[35] T.J. Echtermeyer, L. Britnell, P.K. Jasnos, A. Lombardo, R.V. Gorbachev, A.N. Grigorenko, A.K. Geim, A.C. Ferrari, and K.S. Novoselov, Strong plasmonic enhancement of photovoltage in graphene. Nat. Commun. 2 (2011) 458.

[36] Y. Yao, R. Shankar, P. Rauter, Y. Song, J. Kong, M. Loncar, and F. Capasso, Highresponsivity mid-infrared graphene detectors with antenna-enhanced photocarrier generation and collection. Nano Lett. 14 (2014) 3749-3754.

[37] X. Li, J. Zhu, and B. Wei, Hybrid nanostructures of metal/two-dimensional nanomaterials for plasmon-enhanced applications. Chem. Soc. Rev. 45 (2016) 3145-3187. 
[38] Z. Liu, Y. Gong, W. Zhou, L. Ma, J. Yu, J.C. Idrobo, J. Jung, A.H. MacDonald, R. Vajtai, J. Lou, and P.M. Ajayan, Ultrathin high-temperature oxidation-resistant coatings of hexagonal boron nitride. Nat. Commun. 4 (2013) 2541.

[39] J.D. Caldwell, A.V. Kretinin, Y. Chen, V. Giannini, M.M. Fogler, Y. Francescato, C.T. Ellis, J.G. Tischler, C.R. Woods, A.J. Giles, M. Hong, K. Watanabe, T. Taniguchi, S.A. Maier, and K.S. Novoselov, Sub-diffractional volume-confined polaritons in the natural hyperbolic material hexagonal boron nitride. Nat. Commun. 5 (2014) 5221.

[40] S. Dai, Q. Ma, T. Andersen, A.S. McLeod, Z. Fei, M.K. Liu, M. Wagner, K. Watanabe, T. Taniguchi, M. Thiemens, F. Keilmann, P. Jarillo-Herrero, M.M. Fogler, and D.N. Basov, Subdiffractional focusing and guiding of polaritonic rays in a natural hyperbolic material. Nat. Commun. 6 (2015) 6963.

[41] P. Li, M. Lewin, A.V. Kretinin, J.D. Caldwell, K.S. Novoselov, T. Taniguchi, K. Watanabe, F. Gaussmann, and T. Taubner, Hyperbolic phonon-polaritons in boron nitride for near-field optical imaging and focusing. Nat. Commun. 6 (2015) 7507.

[42] Z. Jacob, I.I. Smolyaninov, and E.E. Narimanov, Broadband purcell effect: Radiative decay engineering with metamaterials. Appl. Phys. Lett. 100 (2012) 181105.

[43] I.S. Nefedov, C.A. Valagiannopoulos, S.M. Hashemi, and E.I. Nefedov, Total absorption in asymmetric hyperbolic media. Sci. Rep. 3 (2013) 2662.

[44] S.A. Biehs, M. Tschikin, and P. Ben-Abdallah, Hyperbolic metamaterials as an analog of a blackbody in the near field. Phys. Rev. Lett. 109 (2012) 104301.

[45] A. Poddubny, I. Iorsh, P. Belov, and Y. Kivshar, Hyperbolic metamaterials. Nat. Photonics 7 (2013) 948-957.

[46] S. Dai, Z. Fei, Q. Ma, A.S. Rodin, M. Wagner, A.S. McLeod, M.K. Liu, W. Gannett, W. Regan, K. Watanabe, T. Taniguchi, M. Thiemens, G. Dominguez, A.H.C. Neto, A. Zettl, F. Keilmann, P. Jarillo-Herrero, M.M. Fogler, and D.N. Basov, Tunable phonon polaritons in atomically thin van der waals crystals of boron nitride. Science 343 (2014) 1125-1129.

[47] Z. Jacob, Nanophotonics: Hyperbolic phonon-polaritons. Nat. Mater. 13 (2014) 10811083.

[48] S. Dai, Q. Ma, M.K. Liu, T. Andersen, Z. Fei, M.D. Goldflam, M. Wagner, K. Watanabe, T. Taniguchi, M. Thiemens, F. Keilmann, G.C.A.M. Janssen, S.E. Zhu, P. Jarillo Herrero, 
M.M. Fogler, and D.N. Basov, Graphene on hexagonal boron nitride as a tunable hyperbolic metamaterial. Nat. Nanotechnol. 10 (2015) 682-686.

[49] A. Kumar, T. Low, K.H. Fung, P. Avouris, and N.X. Fang, Tunable light-matter interaction and the role of hyperbolicity in graphene-hbn system. Nano Lett. 15 (2015) 3172-3180.

[50] M.G. Moharam and T.K. Gaylord, Rigorous coupled-wave analysis of planar-grating diffraction. J. Opt. Soc. Am. 71 (1981) 811-818.

[51] E.N. Glytsis and T.K. Gaylord, Three-dimensional (vector) rigorous coupled-wave analysis of anisotropic grating diffraction. J. Opt. Soc. Am. A 7 (1990) 1399-1420.

[52] L. Li, Use of fourier series in the analysis of discontinuous periodic structures. J. Opt. Soc. Am. A 13 (1996) 1870-1876.

[53] L. Li, Reformulation of the fourier modal method for surface-relief gratings made with anisotropic materials. J. Mod. Opt. 45 (1998) 1313-1334.

[54] E. Popov and M. Nevière, Maxwell equations in fourier space: Fast-converging formulation for diffraction by arbitrary shaped, periodic, anisotropic media. J. Opt. Soc. Am. A 18 (2001) 2886-2894.

[55] C.-H. Lin, K.M. Leung, and T. Tamir, Modal transmission-line theory of threedimensional periodic structures with arbitrary lattice configurations. J. Opt. Soc. Am. A 19 (2002) 2005-2017.

[56] L. Li, Fourier modal method for crossed anisotropic gratings with arbitrary permittivity and permeability tensors. J. Opt. A: Pure Appl. Opt. 5 (2003) 345.

[57] http://zhang-nano.gatech.edu/

[58] Y.-B. Chen and K.H. Tan, The profile optimization of periodic nano-structures for wavelength-selective thermophotovoltaic emitters. Int. J. Heat Mass Transfer 53 (2010) 5542-5551.

[59] P. Yeh, Electromagnetic propagation in birefringent layered media. J. Opt. Soc. Am. 69 (1979) 742-756.

[60] J.M. Zhao and Z.M. Zhang, Electromagnetic energy storage and power dissipation in nanostructures. J. Quant. Spectrosc. Radiat. Transfer 151 (2015) 49-57.

[61] L.P. Wang and Z.M. Zhang, Phonon-mediated magnetic polaritons in the infrared region. Opt. Express 19 (2011) A126-A135. 
[62] N. Engheta, Circuits with light at nanoscales: Optical nanocircuits inspired by metamaterials. Science 317 (2007) 1698-1702.

[63] Y.-B. Chen and F.-C. Chiu, Trapping mid-infrared rays in a lossy film with the berreman mode, epsilon near zero mode, and magnetic polaritons. Opt. Express 21 (2013) 2077120785.

[64] A. Sakurai, B. Zhao, and Z.M. Zhang, Resonant frequency and bandwidth of metamaterial emitters and absorbers predicted by an RLC circuit model. J. Quant. Spectrosc. Radiat. Transfer 149 (2014) 33-40.

[65] L.P. Wang and Z.M. Zhang, Resonance transmission or absorption in deep gratings explained by magnetic polaritons. Appl. Phys. Lett. 95 (2009) 111904.

[66] P.L. Stiles, J.A. Dieringer, N.C. Shah, and R.P.V. Duyne, Surface-enhanced raman spectroscopy. Annu. Rev. Anal. Chem. 1 (2008) 601-626. 


\section{Figure Captions}

Fig. 1 Schematic of the hBN/metal grating hybrid structure, where the Ag grating period, height, and trench width are $\Lambda, h$, and $d$, and the thickness of the $\mathrm{hBN}$ is $d$. The LC circuit model is overlaid on the hybrid structure.

Fig. 2 Illustration of the numerical model for general 2D periodic multilayer structures consisting of anisotropic materials. The structure in Fig. 1 is a special case that has a grating layer in Region II with a periodicity only in the $x$-direction.

Fig. 3 In-plane (ordinary) and out-of-plane (extraordinary) dielectric functions of hBN: (a) the real part and (b) the imaginary part. The two hyperbolic regions are shaded and marked with the corresponding type of hyperbolicity.

Fig. 4 Isofrequency curves for (a) Type-II and (b) Type-I hyperbolic regions. $\mathbf{S}$ represents the Poynting vector and $\beta$ is the angle between $\mathbf{S}$ and the $z$-axis.

Fig. 5 Contour plot showing $\operatorname{Im}(r)$ based on Eq. (17) of a 30-nm-thick suspended hBN film in vacuum near (a) the higher-frequency (Type-II) hyperbolic region and (b) the lower-frequency (Type-I) hyperbolic region. The bright bands indicate high values or poles of $\operatorname{Im}(r)$. The predictions from Eq. (17) are overlaid as broken lines.

Fig. 6 Absorptance spectra of plain Ag gratings and hBN-covered Ag gratings: (a) $h=1.76$ $\mu \mathrm{m}$, (b) $h=2.4 \mu \mathrm{m}$, and (c) $h=0.88 \mu \mathrm{m}$. The default parameters in the present study are $\Lambda=4 \mu \mathrm{m}, b=300 \mathrm{~nm}$, and $d=30 \mathrm{~nm}$. The absorptance spectrum of a suspended 30-nm-thick hBN film is also shown in (a).

Fig. 7 Absorptance contours of (a) plain Ag gratings and (b) hBN-covered Ag gratings in terms of frequency and the grating height. The other parameters are the same as for Fig. 6. The predictions from the LC circuit model are shown as round markers on both plots. The two hyperbolic regions are between the horizontal white lines in (b).

Fig. 8 Local power dissipation density at $v=1436 \mathrm{~cm}^{-1}$ corresponding to the perfectabsorptance peak in Fig. 6(a): (a) Zoomed-in profile showing the details inside the hBN film, (b) The dissipation profile (enlarged by one order of magnitude) of the 
structure. The scale bar for $w$ is in $\mathrm{MW} / \mathrm{m}^{3}$ with an upper limit $1 \mathrm{MW} / \mathrm{m}^{3}$, beyond which $w$ is displayed with white color. Note that the $x$ and $z$ scales are readjusted to show the profile clearly but the propagation angle $\beta$ is distorted.

Fig. 9 Local power dissipation density at $v=818.7 \mathrm{~cm}^{-1}$ corresponding to the high absorptance peak in Fig. 6(b): (a) Zoomed-in profile, (b) The dissipation profile (enlarged by one order of magnitude) of the structure. The scale bar is in $\mathrm{MW} / \mathrm{m}^{3}$ with an upper limit $1 \mathrm{MW} / \mathrm{m}^{3}$, beyond which $w$ is displayed with white color.

Fig. 10 (a) Local power dissipation density at $v=1333 \mathrm{~cm}^{-1}$ corresponding to the near-perfect absorption peak in Fig. 6(c). The scale bar is in $\mathrm{MW} / \mathrm{m}^{3}$ with an upper limit 0.5 $\mathrm{MW} / \mathrm{m}^{3}$, beyond which $w$ is displayed with white color. (b) Local power dissipation density profile of the top $(z=0 \mathrm{~nm})$, the middle $(z=15 \mathrm{~nm})$, and the bottom $(z=30$ $\mathrm{nm})$ of the $\mathrm{hBN}$ film. 


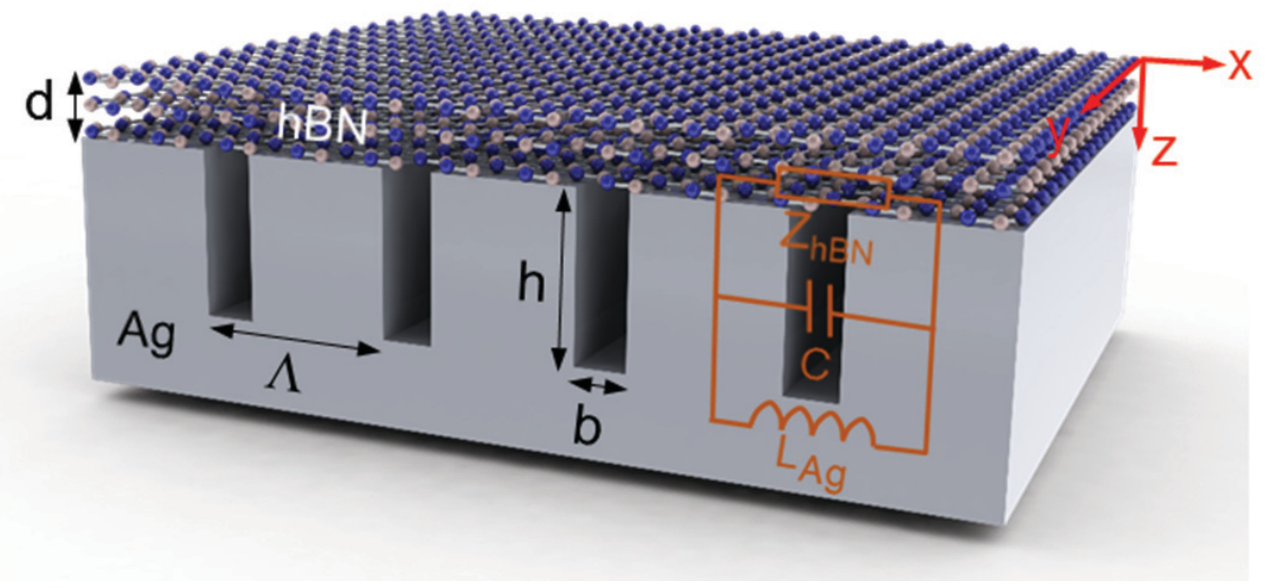

Zhao and Zhang, Fig. 1 


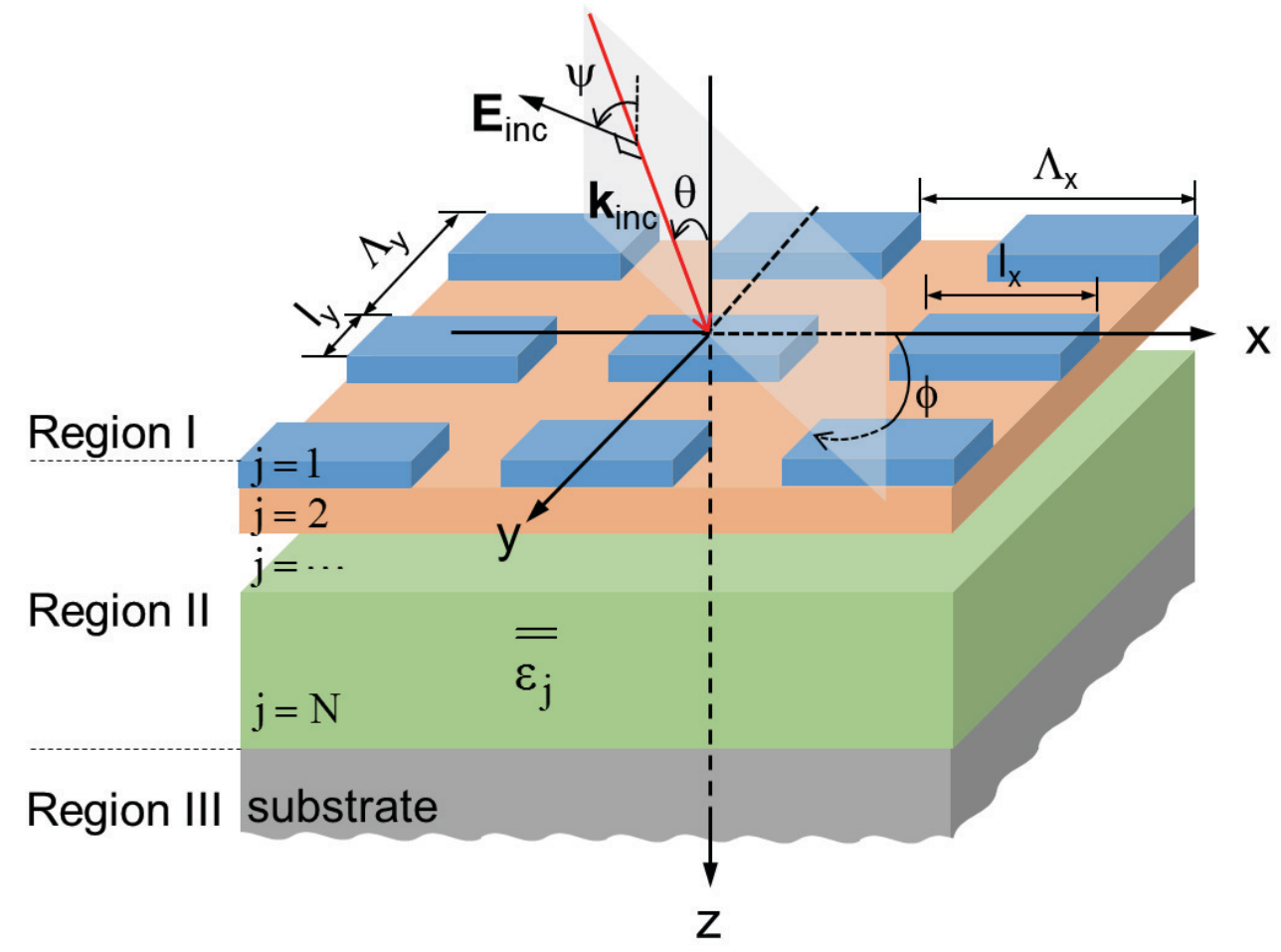

Zhao and Zhang, Fig. 2 

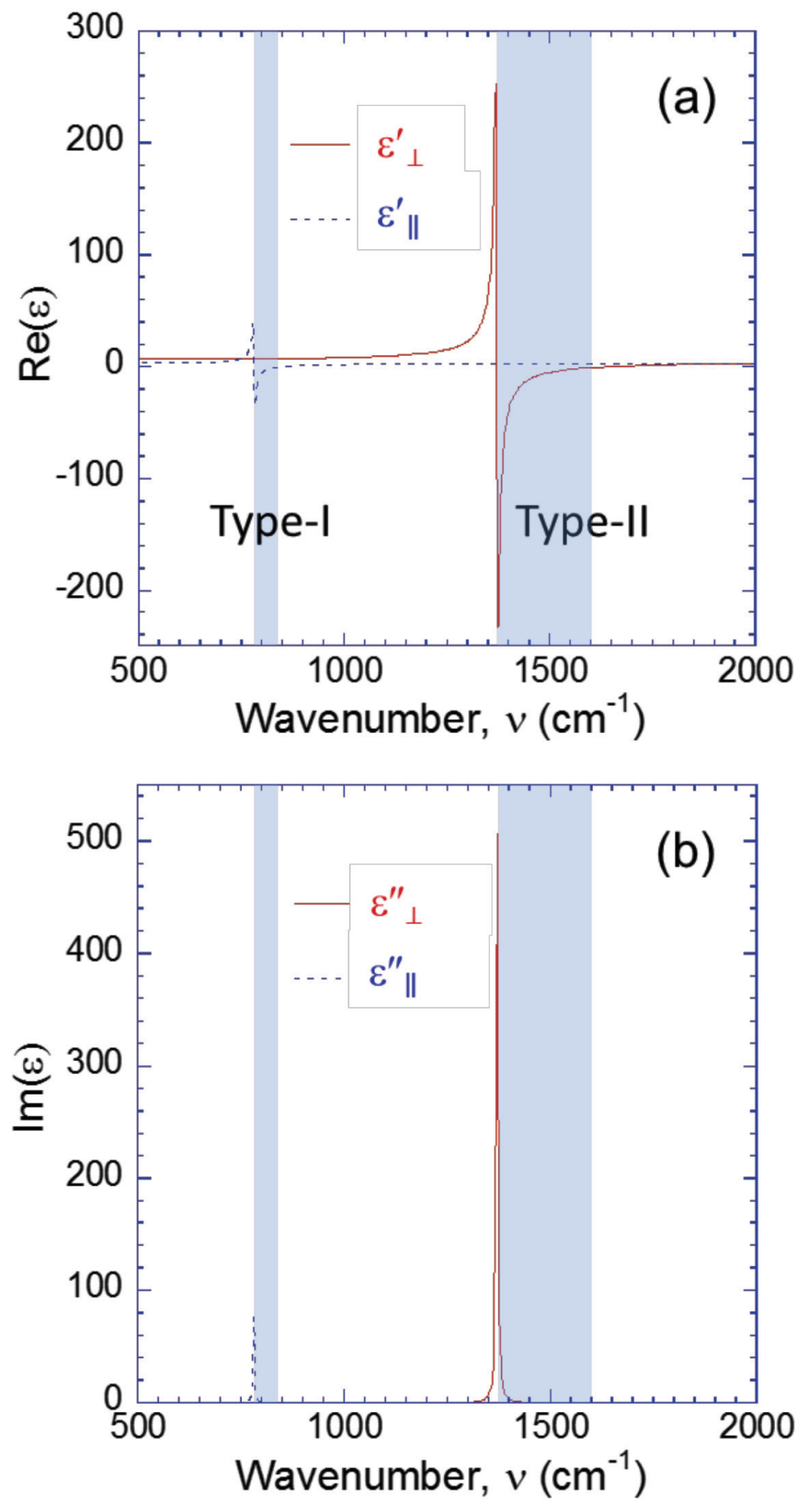

Zhao and Zhang, Fig. 3 


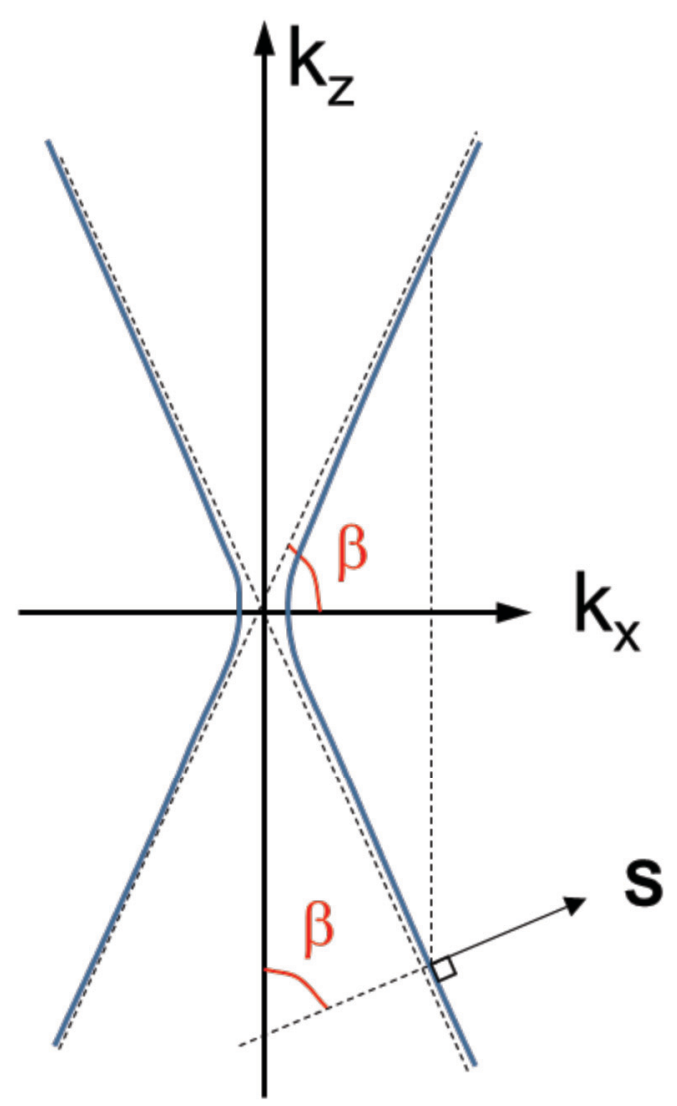

(a) Type-II hyperbolicity

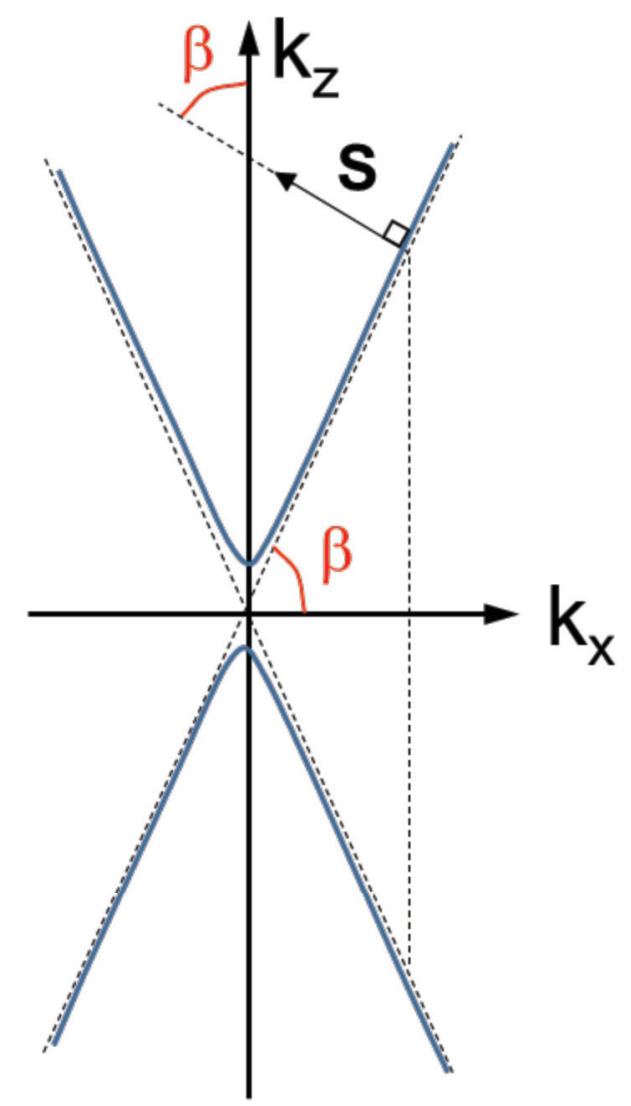

(b) Type-I hyperbolicity

Zhao and Zhang, Fig. 4 


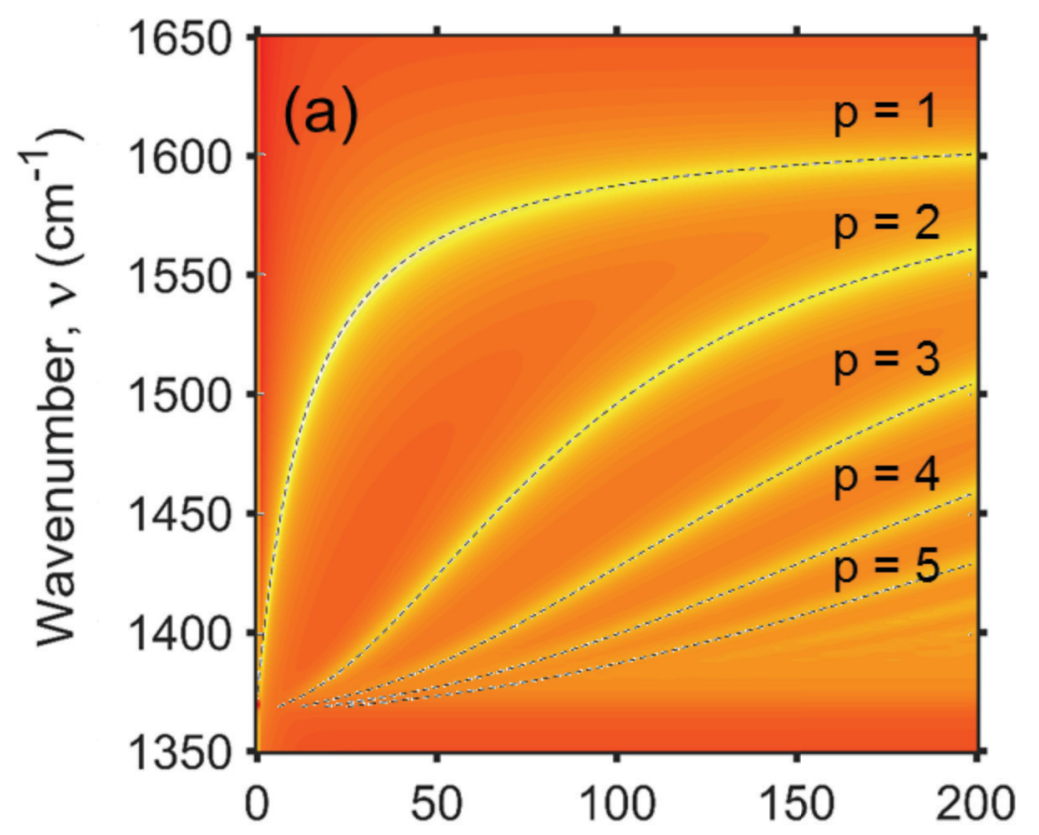

Dimensionless wavevector, $\mathrm{k}_{\mathrm{x}} / \mathrm{k}_{0}$

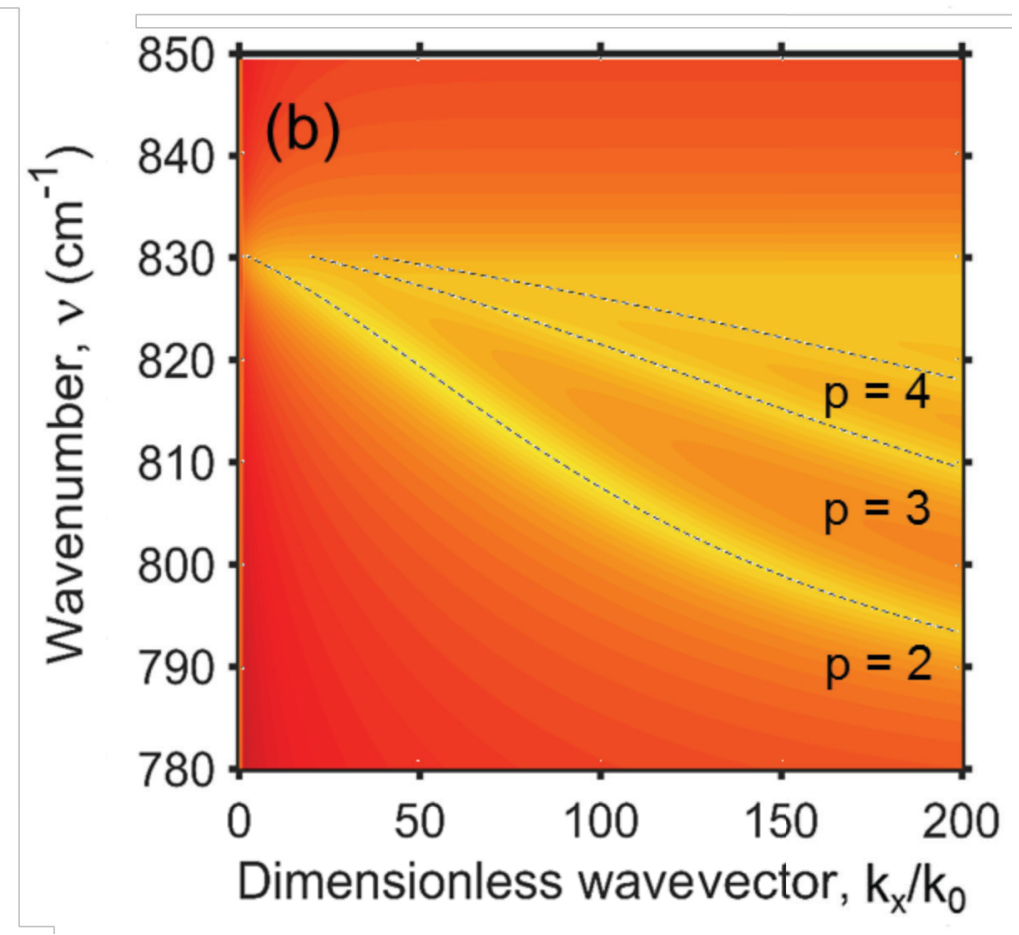

Zhao and Zhang, Fig. 5 

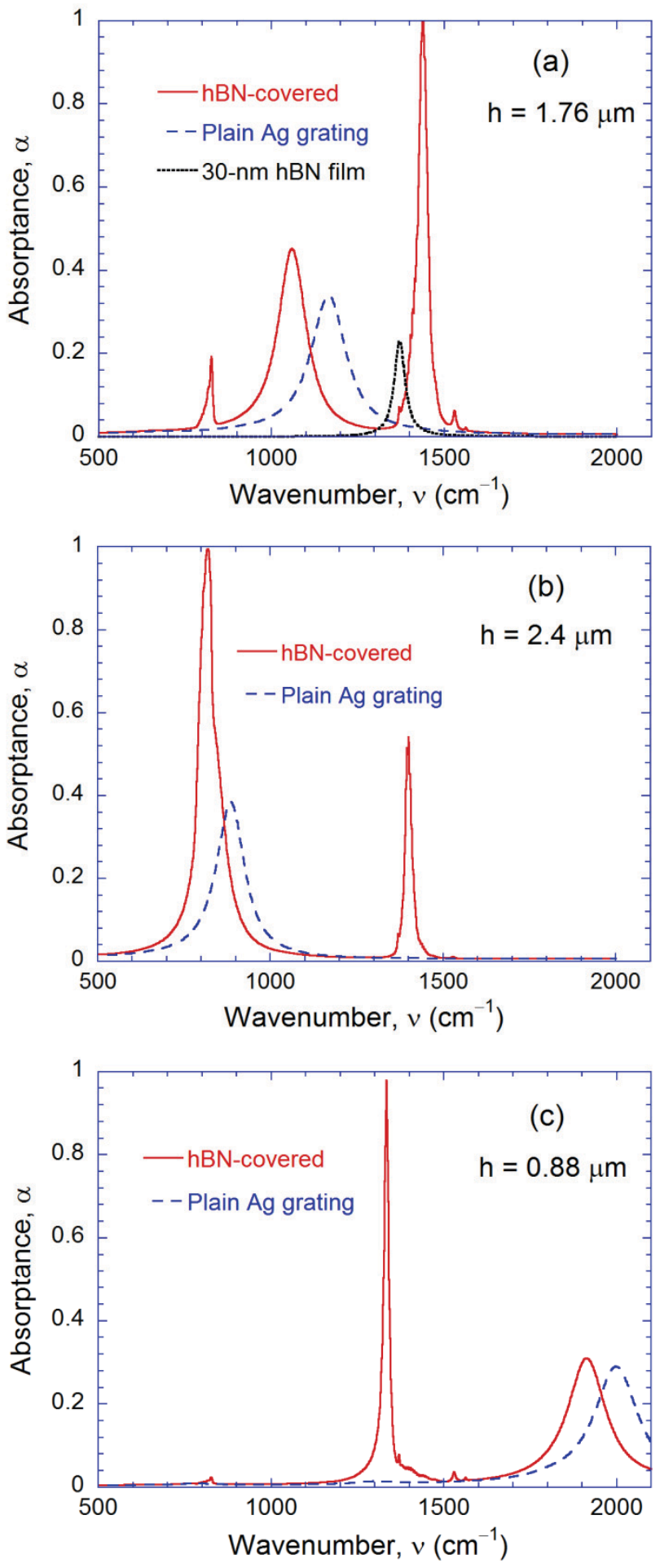

Zhao and Zhang, Fig. 6 


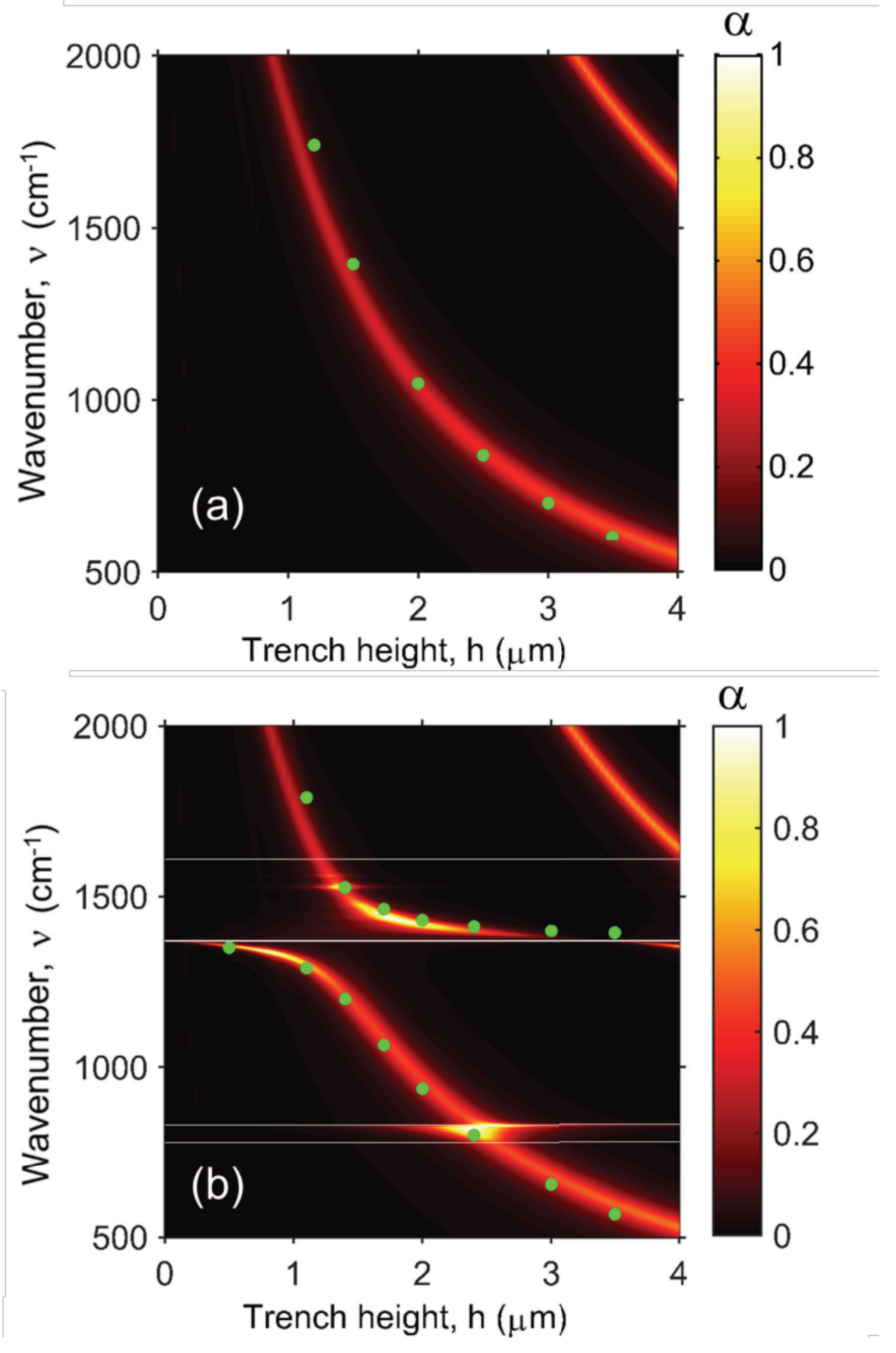


Zhao and Zhang, Fig. 7

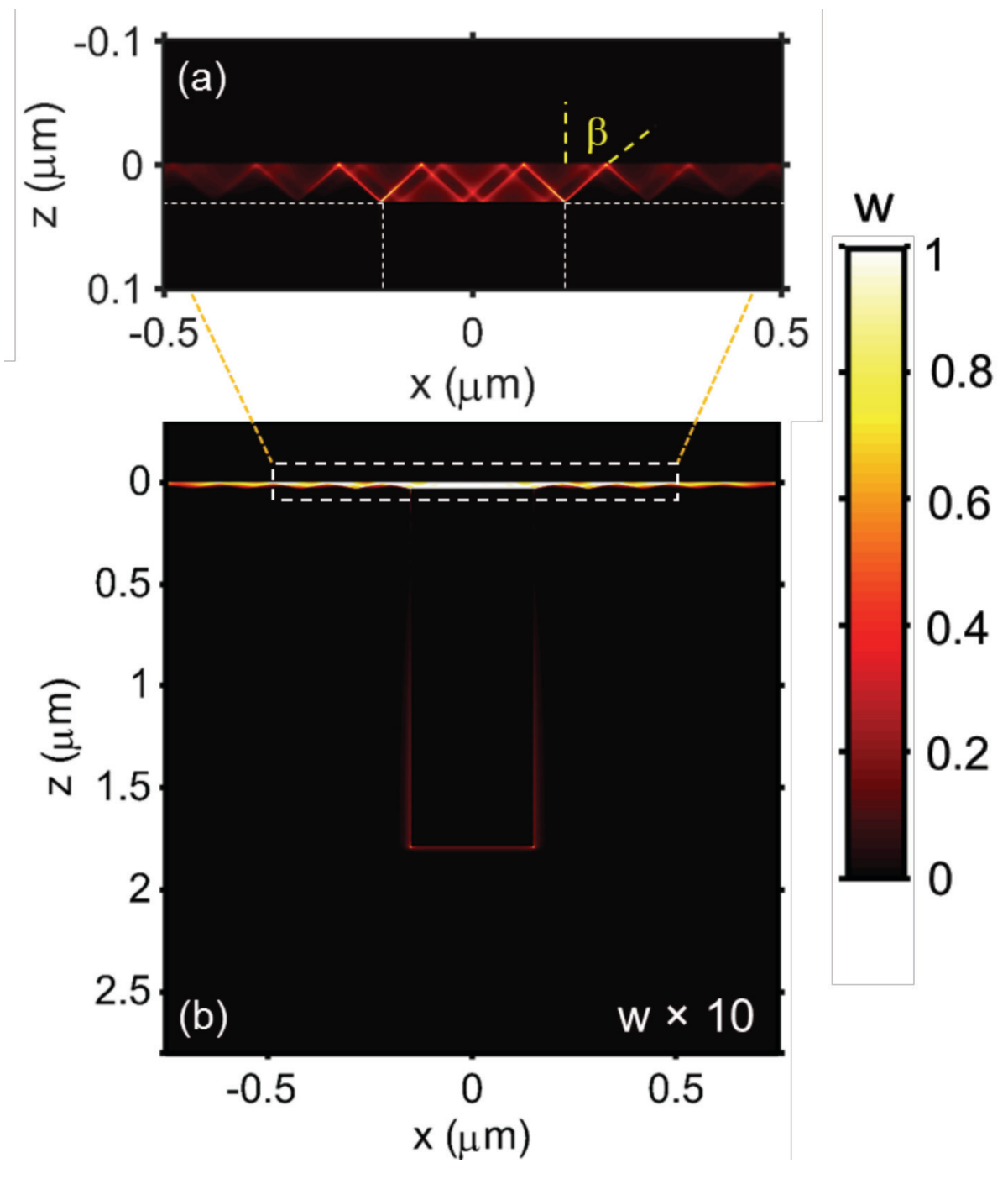


Zhao and Zhang, Fig. 8

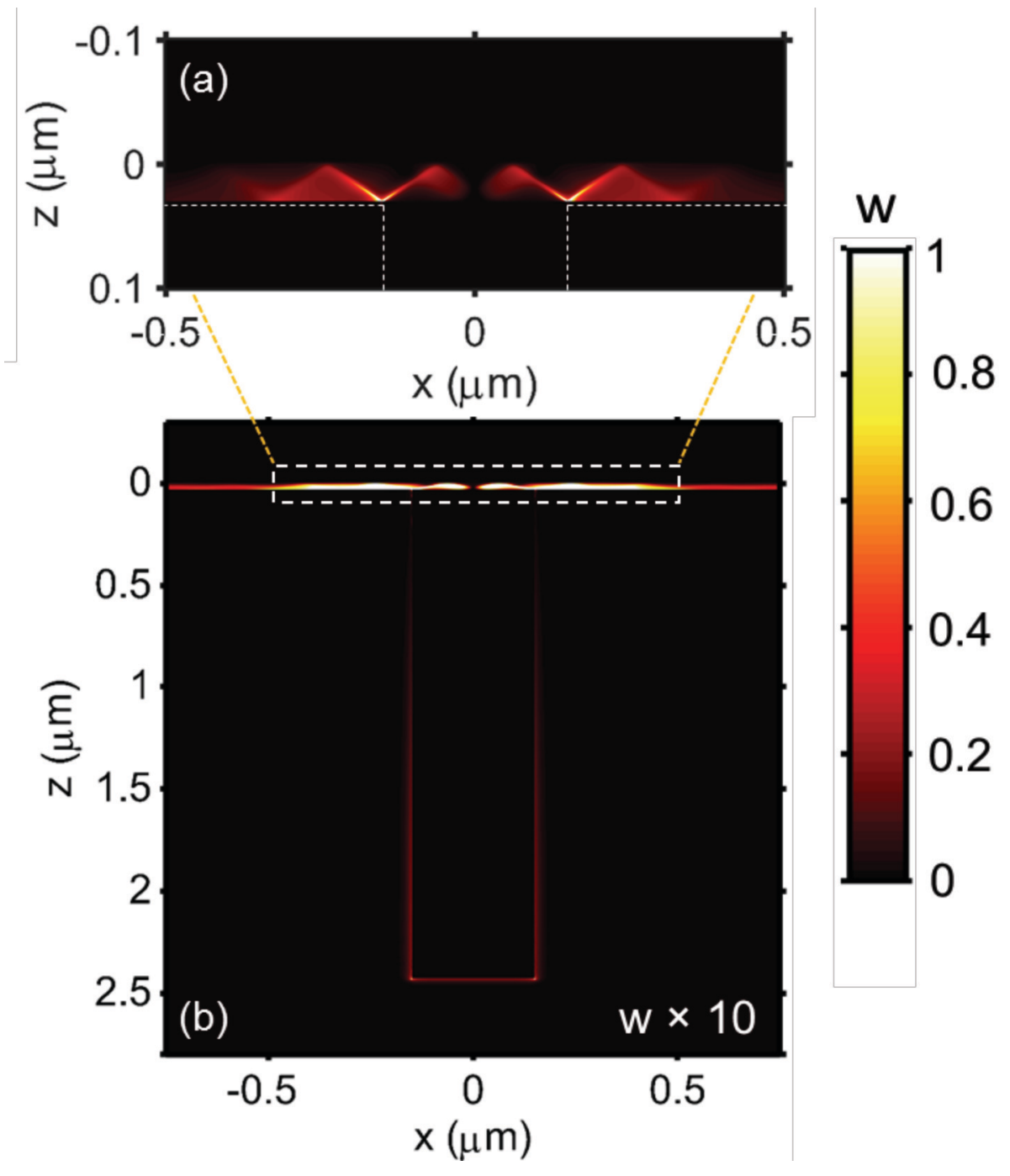

Zhao and Zhang, Fig. 9 

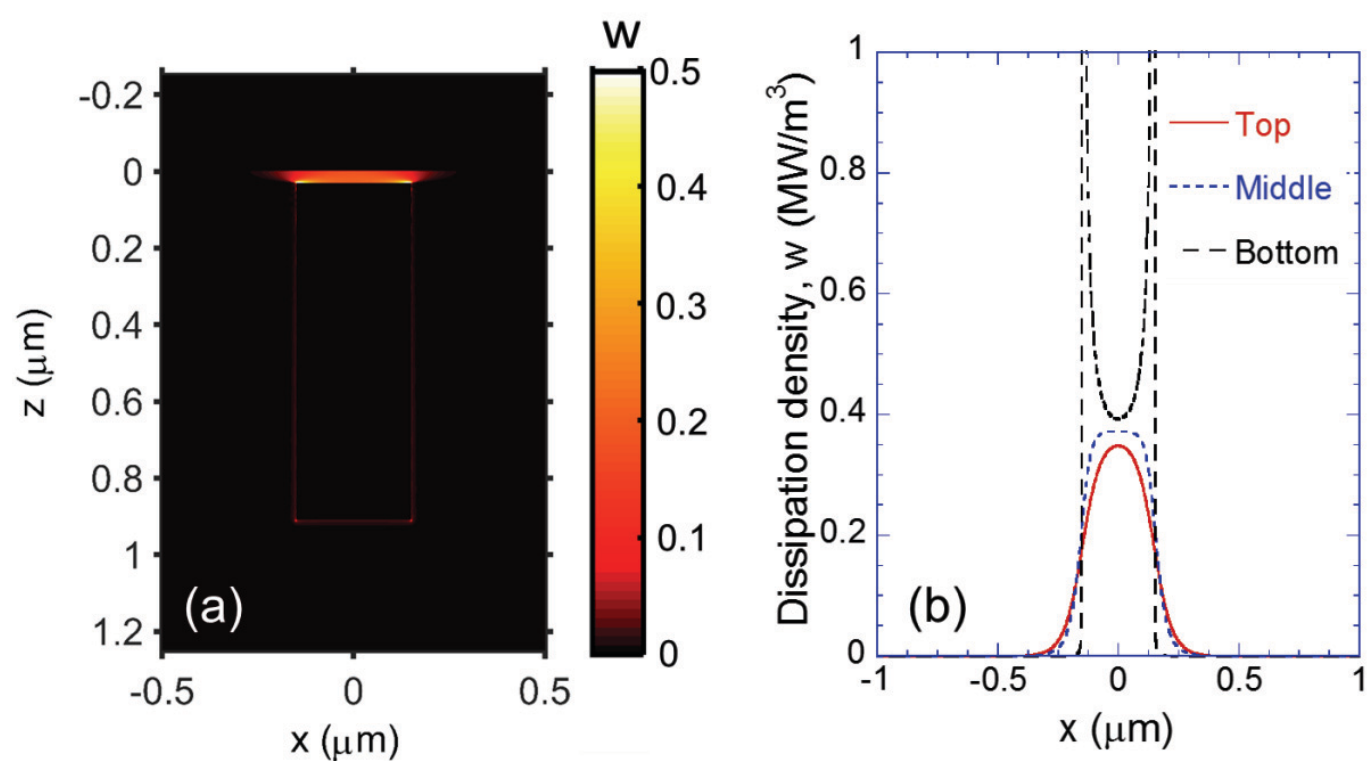

Zhao and Zhang, Fig. 10 